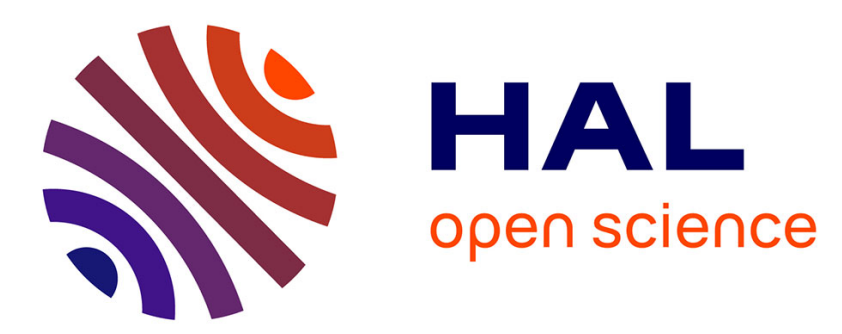

\title{
Experimental and numerical characterization of thin woven composites used in printed circuit boards for high frequency applications
}

\author{
Gautier Girard, Mohamad Jrad, Slim Bahi, Marion Martiny, Sébastien \\ Mercier, Laurent Bodin, David Nevo, Sophie Dareys
}

\section{To cite this version:}

Gautier Girard, Mohamad Jrad, Slim Bahi, Marion Martiny, Sébastien Mercier, et al.. Experimental and numerical characterization of thin woven composites used in printed circuit boards for high frequency applications. Composite Structures, 2018, 193, pp.140-153. 10.1016/j.compstruct.2018.03.037 . hal-02940996

\section{HAL Id: hal-02940996 https://hal.science/hal-02940996}

Submitted on 16 Sep 2020

HAL is a multi-disciplinary open access archive for the deposit and dissemination of scientific research documents, whether they are published or not. The documents may come from teaching and research institutions in France or abroad, or from public or private research centers.
L'archive ouverte pluridisciplinaire HAL, est destinée au dépôt et à la diffusion de documents scientifiques de niveau recherche, publiés ou non, émanant des établissements d'enseignement et de recherche français ou étrangers, des laboratoires publics ou privés. 


\title{
Experimental and numerical characterization of thin woven composites used in printed circuit boards for high frequency applications
}

\author{
Gautier Girard ${ }^{\mathrm{a}}$, Mohamad Jrad ${ }^{\mathrm{a}}$, Slim Bahi ${ }^{\mathrm{a}}$, Marion Martiny ${ }^{\mathrm{a}}$, Sébastien Mercier ${ }^{\mathrm{a}, *}$, Laurent Bodin ${ }^{\mathrm{b}}$ \\ David Nevoc ${ }^{\mathrm{c}}$, Sophie Dareys ${ }^{\mathrm{d}}$ \\ ${ }^{a}$ Université de Lorraine, LEM3 Laboratoire d'Etude des Microstructures et de Mécanique des Matériaux, UMR CNRS 7239, \\ 7 rue Félix Savart 57073 Metz Cedex 03, France \\ ${ }^{b}$ CIMULEC, ZI Les Jonquières, 57365 Ennery, France \\ ${ }^{c}$ Thales Alenia Space, 26 Avenue Jean François Champollion 31100 Toulouse, France \\ ${ }^{d}$ CNES, Centre spatial de Toulouse 18 Avenue Edouard Belin 31401 Toulouse Cedex 9, France
}

\begin{abstract}
The elastic orthotropic behavior of thin woven composites is studied combining a numerical and experimental strategy. For thin materials, in-plane properties are measured by classical tensile tests and digital image correlation. The out-of-plane properties are derived performing finite element simulations at the level of the internal structure of the laminate. Indeed, the glass fiber arrangement in the yarn and the weaving pattern are defined based on microtomography and SEM observations. So a representative unit cell is found. A statistical approach is further proposed to derive the behavior of the warp and fill yarns, since the fiber position may fluctuate between yarns. For the considered laminate, the matrix (resin and ceramic inclusion) behavior is unknown and difficult to measure. Therefore an inverse method is proposed. By comparing with measured in-plane elastic moduli, behaviors of the matrix, of yarns and of the laminate are defined. The present homogenization strategy is exemplified by laminates used in printed circuit boards for high frequency applications. This approach has also been applied to investigate the evolution of the elastic moduli of the laminate with temperature. Those information, usually not available in the literature, are important when dealing with reliability of printed circuit boards during thermal cycles.
\end{abstract}

Keywords: woven composite, mechanical tests, X-ray tomography, orthotropic behavior, numerical homogenization, printed circuit boards

\section{Introduction}

In a context of miniaturization of electronic devices, the dimensions of Printed Circuit Boards (PCB) decrease sensibly with a constant growing interconnection density. The PCB is nowadays not only the carrier of electronic devices but may contain passive or active components (Kpobie et al. [1]). This densification leads manufacturers to the limits of their expertise. In this study, specific material adopted in the bare board is investigated, i.e. no SMD component assembly is considered. The PCB is a multi-layer, multi-material combining dielectric laminates and copper ensuring the conductive pathways. For space or military aeronautic applications, PCBs should withstand a large number of thermal cycles without failure. Materials used in PCB have very different thermomechanical behaviors. In particular, the difference in the coefficient of thermal expansion of copper and composite laminates is the source of some failures (cracks in metallization or in insulation layers, interface problems...). In order to anticipate reliability problems in PCBs, the use of simulation is a necessity, allowing thus to work around the design strategy and avoiding the definition of several unsuccessful prototypes. By simulating different con-

\footnotetext{
* Corresponding author

Email address: sebastien.mercier@univ-lorraine.fr (Sébastien Mercier)
}

figurations, it is possible to determine the most reliable design and thus improve the fatigue life of the PCB [2, 3, 4, 5, 6].

Beforehand, numerical models need to be supplied by precise data. Therefore, the behaviors of all concerned materials have to be precisely determined, namely the elasto-plastic behavior of copper and the complete elastic behavior of laminates. For PCB applications, laminates are usually woven composites, made of one or several glass frames embedded into a resin phase that may contain inclusions. The behavior of laminates or composite plies is orthotropic. For multi-layer PCBs, the thickness of any individual ply is usually a few hundreds of micrometers. Tensile tests in the plane of the laminate allow us to measure in-plane elastic properties. However, the characterization of the mechanical behavior is difficult in the out of plane direction. The idea is to estimate these elastic moduli by means of numerical simulations.

A large majority of laminates used in PCBs are composites made of one or several plain weave glass fabrics coated by a polymer matrix. The weaving pitch, the number of fibers by yarn or the composition of the matrix can vary, depending on the application. Numerous methods have already been developed in the literature to model woven composites: analytical as well as numerical methods.

Several authors have focused on the derivation of analytical models, to obtain the homogenized behavior. As woven fabric composites were gaining technological importance, Ishikawa 
and Chou [7] have presented a model to predict the elastic properties of such materials. The model is also built to describe the non-linear behavior due to the failure of the fabric. The prediction of the elastic behavior is obtained by considering a simplified mosaic representation of the woven composite, in which each cell is assigned a different material, either unidirectional composite or matrix. Classical Laminate Theory (CLT) is adopted to compute the average stiffness of the composite. Naik and Ganesh [8] have proposed to describe precisely the laminate structure from micrograph observations. From the measurements, the laminate unit cell is discretized into slices representing the different components (warp, fill yarns or matrix). This is called the slice array model, where slices are analyzed separately and assembled in parallel or series to obtain bounds for the elastic moduli. In some specific cases, the bounds may be too wide. A second method proposed by Naik and Ganesh [8] is the element array model, in which the slices are subdivided into elements which are analyzed separately and then assembled together. These models are compared to experiments and give good estimates. Sottos et al. [9] have established two analytical models to homogenize the behavior of a plain weave laminate. The equivalent laminate model uses composite cylinder assemblage (Hashin and Rosen [10]) on each yarn and classical laminate theory to get the overall behavior. In the blending model, micromechanical relationships are used to combine the orthotropic warp and fill bundles and give more accurate predictions. Xiong et al. [11] have developed an analytical model by considering a simplified stitch. The volume of fibers is computed and introduced in the simplified model on which computations are done.

Numerical approaches are extensively proposed in the literature. Döbrich et al. [12] have worked on a near micro-scale model. The digital element approach proposed by Wang and Sun [13] is used to reduce the computation time. With this approach, the unit cell is represented with almost all fibers so a two-level homogenization is not needed and the geometry is very realistic. To generate the unit cell, a first simulation has to be performed to get the positions of all fibers. From this work, it is observed that the exact number of fibers does not have to be represented. It has been shown that representing more than 60 fibers per yarn does not affect the predictions for the overall behavior. Fuchs et al. [14] have worked on the homogenization of a PCB (including the copper layer). The behavior of the laminate is computed by a finite element homogenization using the commercial software Digimat. The Mori-Tanaka scheme is used to determine the yarn response. Note that the yarn contains two phases: matrix and fibers. A FE model of the composite representative volume element containing only voxels is generated. Barbero et al. [15], Chen et al. [16] or Jacques et al. [17] also propose numerical homogenization methods for woven composites. The methods are quite similar and first consist on determining the homogenized transverse isotropic behavior of yarns (fibers + matrix), using micromechanical method. A Representative Volume Element (RVE) of the structure is defined and periodic boundary conditions are applied on it. The RVE is subjected to different loadings in order to deduce the homogenized elastic behavior. Barbero et al. [15] assigned a material orientation to the yarns since fiber alignments present some undulation (represented usually by a sine curve) within the weaving pattern. Chen et al. [16] do an averaging along the sine curve to capture the behavior of the yarn. Jacques et al. [17] have implemented the transverse isotropic homogenized yarn behavior without taking the oscillation into account. Bai et al. [18] concentrated on the micro-scale behavior, at the yarn level. They propose to describe the visco-thermo-mechanical behavior of the matrix in order to describe the material deterioration. Green et al. [19] represented a 3D woven composite and homogenized its behavior based on a FE model. An important work has been done on the geometry definition and it has been noticed that a simplified model gives less accurate results than a realistic model (with a geometry closer to the reality). The 3D RVE models for the composite are generated by the software TexGen. Again a unit cell is studied in which the homogenized yarns are considered and periodic boundary conditions (PBC) are applied. This unit cell is discretised with voxels. Since the meshes are identical on opposite faces of the RVE, implementation of the PBCs is straightforward. In addition, a voxel may contain more than one phase but by construction, the material response at the level of the voxel corresponds to the one of the dominant phase (in volume). With such strategy of voxel based finite elements, the presence of resin between crossing yarns is avoided. In a classical FE model, the remaining volume of matrix phase between yarns may induce the presence of small and distorted elements, leading to numerical singularities. The behavior of the yarns is considered as transversely isotropic and is homogenized using a finite element analysis. The authors still observed a small difference in predictions between experiments and numerical simulations. The origin of the discrepancy is expected to originate from the voxelisation of the real microstructure.

Different homogenization methods for the yarns are investigated in the literature. One can cite the Mori-Tanaka method, the self-consistent scheme, the three phase approach, the composite cylinder assemblage, or the finite element homogenization based on the description of a RVE with fibers and matrix, as used by Green et al. [19] or Srbová et al. [20]. In Chen et al. [16] or in Abaimov et al. [21], different homogenization methods are compared, but over a certain volume fraction of fibers, the quality of the predictions is reduced. In our field of interest, the volume fraction of fibers is larger than $60 \%$. Furthermore, as observed in most of the literature, the matrix behavior or more generally the behaviors of all phases are known and their elastic properties well established. In the PCB industry, for very specific applications like for high frequency applications, information concerning the matrix behavior is difficult to obtain and its composition is usually considered confidential by the manufacturer.

To overcome these difficulties, we propose a multistep homogenization approach to derive the elastic behavior of the yarns and the laminate. The novelty is that the homogenization proceeds without knowing a priori the matrix behavior. Indeed an inverse method, based on the comparison between experiments and numerical predictions for the in-plane elastic properties is adopted so that the matrix behavior is a direct outcome 
of our model. The paper is organized as follows. In section 2 the material characterization is described. This includes the measurement of in-plane elastic properties and the precise geometrical description of the laminate. Section 3 explains the homogenization procedure starting from analytical and numerical procedures at the level of the yarns in section 3.2 and leading to the homogenization of the woven composite in section 3.3. The geometry generation, the periodic boundary conditions implementation and the finite element model leading to the elastic properties of the laminate are carefully presented. Finally in section 3.4 , the inverse method used to perform the homogenization without knowing the matrix behavior beforehand is developed and some estimates for the elastic moduli at different temperatures are provided. Such information is rarely available in the literature.

\section{Material characterization}

In this section, a precise material characterization is proposed. The in-plane elastic behavior will be determined and the internal structure of the material will be accurately defined by a combination of images from micro-tomography and scanning electron microscopy devices.

\subsection{Elastic properties}

Elastic properties in the plane of the laminate are first obtained via an electrodynamic tensile machine Instron E3000 equipped with an environmental chamber. The chamber can be heated up to $300^{\circ} \mathrm{C}$, cooled down below $-60^{\circ} \mathrm{C}$ with liquid nitrogen. The front door of the chamber is designed with a heated window so that the tensile test can be video-recorded. This will further permit the determination of strain maps by digital image correlation (DIC). This contactless strain measurement technique is not disturbing the experiment. Other strain measurement technologies like clip-on extensometer, could not have been adopted for thin specimens (as considered in the present work).

The tested material is a laminate designed for high frequency applications, with a thickness of $h=309 \mu \mathrm{m}$. The composite is made of a woven glass fabric and the hydrocarbon (not PTFE) resin contains ceramic particles. Owing to the structure of the material, its elastic behavior is orthotropic. The corresponding Hooke's law can be classically written with the Voigt notation as :

$$
\left\{\begin{array}{c}
\varepsilon_{11} \\
\varepsilon_{22} \\
\varepsilon_{33} \\
2 \varepsilon_{12} \\
2 \varepsilon_{13} \\
2 \varepsilon_{23}
\end{array}\right\}=\left[\begin{array}{cccccc}
\frac{1}{E_{1}} & -\frac{\nu_{12}}{E_{1}} & -\frac{\nu_{13}}{E_{1}} & 0 & 0 & 0 \\
-\frac{\nu_{12}}{E_{1}} & \frac{1}{E_{2}} & -\frac{\nu_{23}}{E_{2}} & 0 & 0 & 0 \\
-\frac{\nu_{13}}{E_{1}} & -\frac{\nu_{23}}{E_{2}} & \frac{1}{E_{3}} & 0 & 0 & 0 \\
0 & 0 & 0 & \frac{1}{G_{12}} & 0 & 0 \\
0 & 0 & 0 & 0 & \frac{1}{G_{13}} & 0 \\
0 & 0 & 0 & 0 & 0 & \frac{1}{G_{23}}
\end{array}\right]\left\{\begin{array}{l}
\sigma_{11} \\
\sigma_{22} \\
\sigma_{33} \\
\sigma_{12} \\
\sigma_{13} \\
\sigma_{23}
\end{array}\right\}
$$

In the PCB industry, the convention usually sets the warp direction as $x$ and the fill direction as $y$. This is also adopted in the present work. Tensile tests have been performed first at room temperature on specimens with $0^{\circ}, 45^{\circ}$ and $90^{\circ}$ orientation (with respect to the $x$ direction), leading to the determination of the elastic moduli $E_{1}, E_{2}, \nu_{12}, G_{12}$ (see classical handbook on composite materials for instance Jones [22]). Table 1 provides the corresponding measured values and compares with the supplier's data. It has to be noticed that the elastic properties given in the datasheet for a given material are usually valid for a specific glass fabric. But the material is commercially available with various standard thicknesses (from $0.203 \mathrm{~mm}$ to $1.524 \mathrm{~mm}$ ) and may contain different woven glass fabrics. As a consequence, the supplier data may not be appropriate for the thickness considered in this work. The mechanical characterization has also been carried out at various temperatures in the range $\left[-55^{\circ} \mathrm{C} ;+125^{\circ} \mathrm{C}\right]$, the temperature range that the $\mathrm{PCB}$ may experience during thermal cycles compulsory for the qualification process to space standard. In fact, such temperature dependency of the elastic properties is usually not provided by the supplier, while this information is of primary importance to investigate by finite element calculations the reliability of the PCB during thermal cycles. Data at different temperatures are presented later in the paper, see Fig. 14.

\begin{tabular}{lllll}
\hline & $E_{1}[\mathrm{GPa}]$ & $E_{2}[\mathrm{GPa}]$ & $\nu_{12}[-]$ & $G_{12}[\mathrm{GPa}]$ \\
\hline experimental measurements & 18.53 & 20.37 & 0.168 & 5.78 \\
datasheet values & 25.510 & 26.889 & - & - \\
\hline
\end{tabular}

Table 1: In-plane elastic properties at room temperature. Comparison between measurements and datasheet values for the studied laminate designed for high frequency applications. Difference originates from the glass fiber fabric of the tested laminate which is not similar to the one characterized by the supplier and whose properties are reported in the datasheet.

The out of plane elastic moduli cannot be measured by classical tensile machines, owing to the small sample thickness. As proposed in the literature, estimates will be provided by developing a salient multi-scale approach combining Mori-Tanaka scheme and Finite Element Model, see Section 3.

\subsection{Microstructure definition}

In order to estimate the out-of-plane components, a numerical approach will be proposed in the next sections. So beforehand, it is important to precisely define the laminate microstructure. Indeed, the plain weave fabric structure is not unique and may vary depending on the thickness of the composite material. The proposed measurements will let us define the geometrical features of a Representative Volume Element (RVE). In section 3.3, a Finite Element Model (FEM) on the real microstructure will provide precise determination of the orthotropic elastic response of the composite.

To describe accurately the laminate structure, measurements have been performed by X-ray microtomography (with a voxel size of $1.7 \mu \mathrm{m}$ ), on a "EasyTom" from RX Solutions, in LEM3. Figure 1 presents a microtomography scan (three orthogonal views) which permits to observe the woven glass fabric and the resin; the process being nondestructive. As mentioned in the datasheet of the supplier, the resin is filled with a large volume content of ceramic particles. In our approach, the resin and the filler content will be considered as the matrix phase. The fill and warp fiber bundles interlace with each other in an orthogonal way. We also observe that the laminate is made of three 
identical superimposed glass fabrics. Based on microtomography, some geometrical features can be determined: the gap between two adjacent yarns $\left(g_{w}\right.$ and $\left.g_{f}\right)$ and the period of the yarn undulation $\left(2 b_{f}\right.$ and $\left.2 b_{w}\right)$.
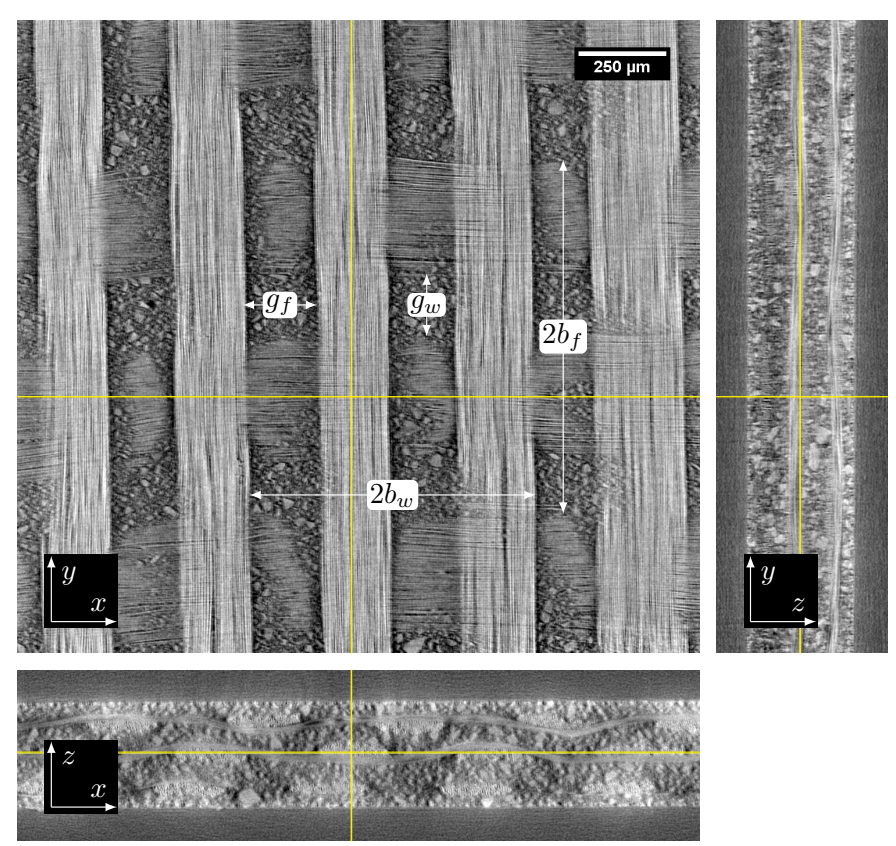

Figure 1: Observation of the laminate by X-ray microtomography. Three orthogonal views are represented and some geometrical measurements of the RVE are drawn. Note that the $x$ direction refers to the warp direction. It is clearly seen that the warp and fill yarns are perpendicular. The matrix is filled with a large volume content of ceramic inclusions.

Similarly to the work of Naik and Ganesh [8], a 3D model accounting for the actual yarn cross-section geometry, the gap between two adjacent yarns and undulation amplitude will be proposed owing to the observations at the microstructure level. Microtomography shows that the cross section of a yarn is similar all along the weaving pattern. For the material considered in the present work, the warp and fill yarns have different crosssections. It is also observed that the warp and fill yarns are in contact when they cross each other, i.e. the shape of the cross section of a warp yarn fits the sinusoidal shape of the crossing fill yarn (see the schematic view on Fig. 2).

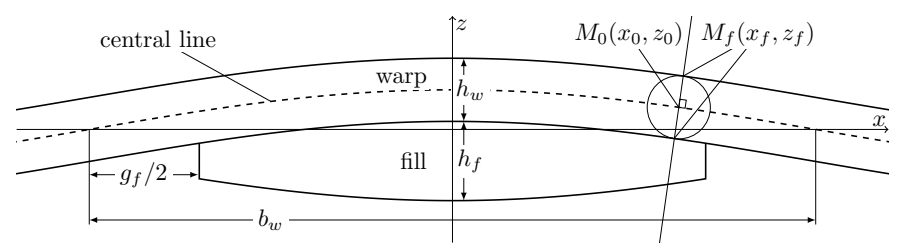

Figure 2: Layout of the cross section of the fill yarn, corresponding to a view in the plane $(x, z)$ of Fig. 1. The shape of the yarn is directly evaluated from the knowledge of the undulation which has been measured by X-ray tomography. $h_{f}$ and $h_{w}$ refers to the yarn thickness, $b_{w}$ to the half size of the RVE in the warp direction and $g_{f}$ the gap between two adjacent fill yarns.

In the warp direction, the undulation of the central line of the yarn is mathematically represented by a sinusoidal curve, see Naik and Ganesh 1992 [8], Chen et al 2014 [16]:

$$
z_{0}=f\left(x_{0}\right)=\frac{h_{f}}{2} \cos \left(\frac{\pi x_{0}}{b_{w}}\right)
$$

where $x_{0}$ and $z_{0}$ are the coordinates of a material point $M_{0}$ lying on the central line of the yarn, $h_{f}$ the thickness of the fill yarn and $b_{w}$ the pitch of the warp yarn. Note that $2 b_{w}$ is the period of an undulation in the warp direction and will correspond to the size of the RVE in this direction. To describe the fill direction undulation, the same equation (2) is used by replacing $h_{f}$ by $h_{w}$ and $b_{w}$ by $b_{f}$.

Since warp and fill yarns are in contact when they cross (there is no resin in between), the outer frontier of the cross section of a yarn can be computed directly from the knowledge of the undulation curve, as follows. Point $M_{f}\left(x_{f}, z_{f}\right)$ on the frontier of the fill yarn cross section should be located at a constant distance from the central line of the warp yarn that it is crossing; this distance being $h_{w} / 2$ (half thickness of the warp yarn). In addition, we assume that the point $M_{f}$ is also placed on the perpendicular line to the cosine function (see Fig. 2). Based on these two requirements, for a point $\left(x_{0}, z_{0}\right)$ on the central line of the warp yarn, the coordinates of the corresponding point $M_{f}\left(x_{f}, z_{f}\right)$ on the border point of the fill yarn are found as the solution of the following system:

$$
\left\{\begin{array}{c}
\left(x_{f}-x_{0}\right)^{2}+\left(z_{f}-z_{0}\right)^{2}=\left(\frac{h_{w}}{2}\right)^{2} \\
z_{f}=-\frac{x_{f}-x_{0}}{f^{\prime}\left(x_{0}\right)}+z_{0}
\end{array}\right.
$$

The system has two solutions $\left(x_{f}, z_{f}\right)$, as seen of Fig.2. We select the solution leading to a point on the frontier of the fill yarn:

$$
\left\{\begin{array}{l}
x_{f}= \pm \frac{h_{w}}{2 \sqrt{1+f^{\prime}\left(x_{0}\right)^{-2}}}+x_{0} \\
z_{f}= \pm \frac{h_{w} f^{\prime}\left(x_{0}\right)^{-1}}{2 \sqrt{1+f^{\prime}\left(x_{0}\right)^{-2}}}+z_{0}
\end{array}\right.
$$

with

$$
f^{\prime}\left(x_{0}\right)=-\frac{h_{f} \pi}{2 b_{w}} \sin \left(\frac{\pi x_{0}}{b_{w}}\right)
$$

As observed on microtomography scans (Fig. 1) and as depicted in Figs. 2 and 3, a gap $g_{f}$ exists between two adjacent fill yarns. Therefore, the frontier of the cross section of a yarn is defined based on the parametric curve (4), its symmetric part and in addition by shortening the tips, see Fig. 2. This defined shape will be extruded in the 3D model to represent a yarn, following the undulation curve.

The microtomography scans provide a first set of geometrical distances, see Fig. 3. Nevertheless, the precise knowledge of the geometrical set is needed for the $3 \mathrm{D}$ model of the fabric. To increase our confidence in the geometrical quantities defining the microstructure of the laminate, complementary observations on yarns and fibers constituting the composite have been done with use of a Scanning Electron Microscope (SEM 


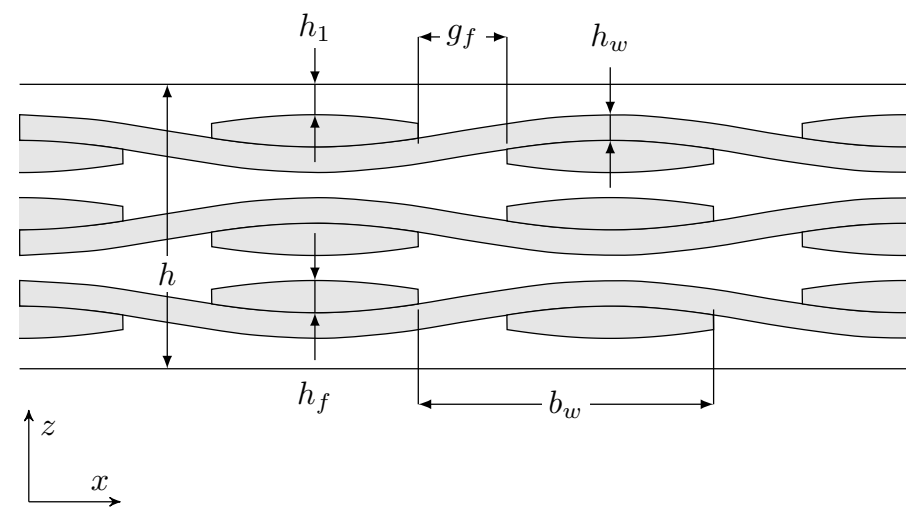

Figure 3: Layout of the weaving pattern, corresponding to a view in the plane $(x, z)$ of Fig. 1. X-ray microtomography provides a first estimate of distances. Scanning electron microscopy observations will confirm these values, as reported in Table 2 .

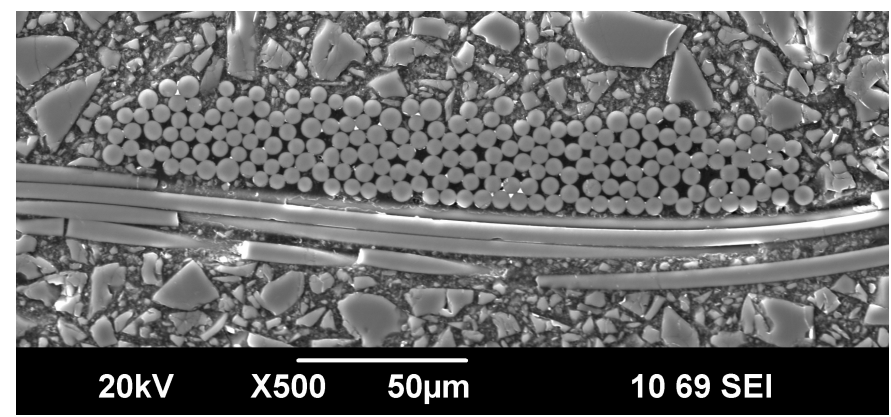

Figure 4: Yarn observation by scanning electron microscopy. Ceramic inclusions of different sizes and shapes can be observed. It is also clear that the radii of fibers are not homogeneous. The surface is polished and a thin layer of black carbon is deposited on the surface for SEM observation.

Zeiss) (Fig. 4). The SEM images will be analyzed and will confirm the global shape of a yarn (Fig. 5). For each SEM image of a yarn, the center and radius of all fibers are detected by a dedicated Matlab program, developed for this work. Note that each yarn contains around two hundred fibers. By repeating this operation on several tens of images, one can superimpose all data on one single plot as presented in Fig. 5. All detected fibers are displayed with some level of transparency and the opacity gets more important as several fibers are superimposed. In that way, as the number of analyzed images increases, the exact shape of the mean yarn is revealed, see Fig. 5. The horizontal extent of the yarn $\left(b_{w}-g_{f}\right.$ for fill yarns, see Fig. 3$)$ and the yarn thickness ( $h_{f}$ for fill yarns) are deduced for each SEM image by evaluating the position of the fibers which are located at the extremities of the yarn. By averaging these values on all images, one gets the mean dimensions of the yarn. The parameter $b_{w}$ being evaluated from microtomography scans and based on the measured $b_{w}-g_{f}$ and $h_{f}$ values, it is possible to plot the contour of the yarn section derived from the Eqs. (4) and (5). This corresponding average yarn cross-section is displayed on Fig. 5 with the solid line. One can see that the proposed strategy reproduced accurately the average shape of the yarn. It was shown that data provided by SEM were consistent with microtomography predictions. But owing to the resolution of both devices, in the following, we will adopt geometrical values captured based on SEM images. Table 2 lists all geometrical features necessary for the 3D definition of the woven glass fabric. Combining data of Table 2 and Eqs. (4) and (5), the frontier of the warp and fill yarns are defined. As observed in Fig. 5, the warp and fill yarns have different cross-section but also different volume content of glass fibers. For each SEM image, the fiber volume fraction in the yarn can be computed by dividing the sum of the areas of all fibers by the area of the mean yarn (as defined previously). The averages of the fiber volume fraction on all images are $62 \%$ for the warp direction and $67 \%$ for the fill one.

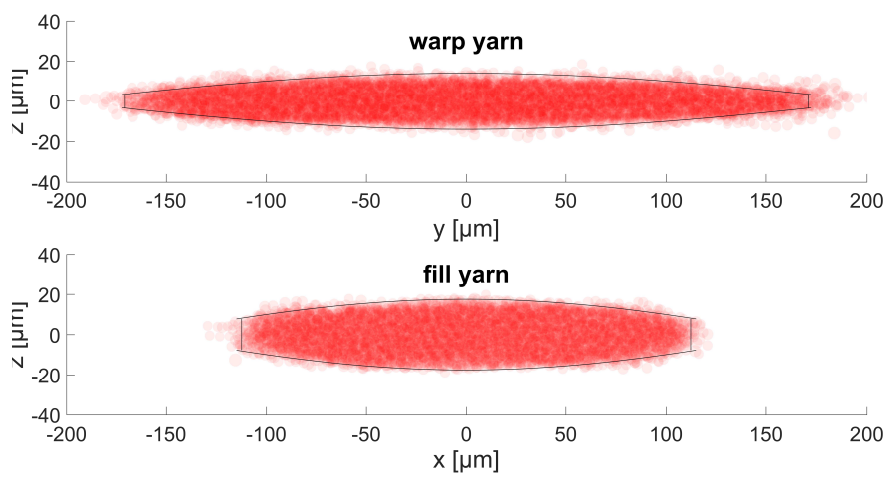

Figure 5: Shape of the fill and warp yarns after observation by SEM. A statistical analysis on several tens of yarns leads to the definition of a mean shape that will be used for numerical simulations. The solid line reproduces the outer surface of the yarn, as defined by Eq. (4). A good agreement between the locations of thousands fibers and the mathematical description of both yarns is observed. The warp yarn is larger than the fill yarn while the fill yarn is thicker. The volume fraction of fibers is $62 \%$ (resp. 67\%) for the warp yarn (resp. fill yarn).

\begin{tabular}{llllllll}
\hline$h$ & $h_{1}$ & $h_{f}$ & $b_{f}$ & $g_{f}$ & $h_{w}$ & $b_{w}$ & $g_{w}$ \\
\hline 309 & 33.0 & 35.0 & 405 & 96.3 & 27.9 & 321 & 62.9 \\
\hline
\end{tabular}

Table 2: Geometrical quantities characterizing the internal structure of the studied laminate (in $\mu \mathrm{m}$ ). One may refer to Figs 1 and 3 for illustration.

The precise observation of SEM images representing a large number of warp and fill yarns provides additional information which is not often present in the literature. Indeed, the fiber detection by the Matlab program provides also the fiber radii. From these measurements, the mean fiber radius can be calculated and the radii distribution can be evaluated. Fig. 6 shows the experimental probability density function for the fiber radius and the corresponding fitted curve with a Burr distribution:

$$
p d f_{\text {Burr }}(r, c, k, \alpha)=\frac{c k}{\alpha}\left(\frac{r}{\alpha}\right)^{c-1}\left[1+\left(\frac{r}{\alpha}\right)^{c}\right]^{-k-1}
$$

The parameters for the Burr distribution are: $c=41.935, k=$ 0.366 and $\alpha=2.43 \mu \mathrm{m}$. It should be mentioned that for the considered material, the fiber radius distribution is identical for 
the warp and fill yarns. The mean radius is $2.57 \mu \mathrm{m}$ and the standard deviation is $0.20 \mu \mathrm{m}$. Fibers have radius in the range $[2 \mu m ; 3.5 \mu m]$. These information will be adopted next for the definition of the homogenized response of the yarn. It will allow us to integrate the influence of the fiber radius distribution on the effective elastic properties. In most studies of the literature, the radius of all fibers is assumed identical. Owing the precise characterization of the microstructure (geometrical parameters of the RVE, cross-section of each yarn, amplitude of the undulation, volume fraction of fibers in the fill and warp yarns, radius distribution of fibers), the $3 \mathrm{D}$ model will be closely representative of the studied laminate.

\section{Homogenization of a woven composite}

\subsection{Definition of the Representative Volume Element and ho- mogenization strategy}

From section 2.2 on experimental characterization, a RVE of extent $2 b_{w} \times 2 b_{f} \times h$ is defined. The numerical model representative of the RVE is built on Abaqus using python scripts with the following steps. The shape of the yarn cross-section, defined by Eq. (4), has been drawn in an Abaqus sketch and extruded along the undulation curve (2). Since warp and fill yarns have different cross-sections, they are generated separately, duplicated and placed to reproduce the glass fabric. Finally, the numerical model of the RVE is available for further analysis, see Fig. 12.

From microtomography, the composite material has a complex structure and is made of three different materials: glass for the fibers, ceramic material for the inclusion and hydrocarbon polymer for the resins. Except for the glass fibers which are made of E-glass, none of the elastic properties of the constituents are known.

In the present work, the prediction of the behavior of the laminate is performed at the meso-scale through a two step homogenization procedure in association with an inverse method. First the behavior of the yarns is homogenized from an assembly of unidirectional glass fibers and a resin. Computations have to be done for both the warp and fill directions since the volume fractions of fibers are different. As a consequence, the behavior of the two homogenized warp and fill materials is obtained. Nevertheless, due to lack of data, the behavior of the resin phase present in each yarn is unknown and will be estimated in a next section. In a second step, a finite element model at the level of the RVE is performed. At that stage, the RVE contains three different materials, namely the matrix phase (hydrocarbon resin + ceramic inclusion), the warp and fill materials. Since the behavior of the resin in each yarn and of the matrix phase cannot be measured, then an inverse method will be adopted. The subsequent steps leading to the predictions of orthotropic behavior of the composite material are described in the following of this section.

\subsection{Yarn homogenization}

In the laminate studied here, around 200 fibers can be found in a yarn and the detailed representation in a numerical model would lead to huge computation time. Some attempt was proposed by Döbrich et al. [12]. They have derived the homogenized behavior of a composite with woven glass fabric, based on a numerical model where the fiberglasses were meshed. Nevertheless, due to the large computation costs, the number of fibers represented in a yarn was limited. In addition, in their work, the matrix phase was homogeneous. In the present work, such full numerical strategy is not adopted for several reasons. Here, the matrix phase is filled with a large content of inclusions. Creating a precise model with an accurate description of the microstructure would impose to consider both fibers and also inclusions in the matrix. In addition, the positions of all fibers are varying from yarns to yarns, as observed in Fig. 5. So instead of developing a RVE with fibers and inclusions, we propose to develop a statistical strategy for the definition of the yarn behavior, by testing a large number of configurations.

A first homogenization stage is conducted to define the behavior of yarns. With more than 200 fibers, a yarn is supposed to have a transverse isotropic behavior described by the relation (7):

$$
\underline{\underline{\varepsilon}}=\underline{\underline{\underline{S}}}: \underline{\underline{\sigma}}
$$

where $\underline{\underline{S}}$ stands for the elastic compliance tensor. With the Voigt notation, introducing the engineering moduli (subscripts $A$ and $T$ standing for axial and transverse properties respectively), and considering that fibers are aligned along direction 1 , the elastic law becomes:

$$
\left\{\begin{array}{c}
\varepsilon_{11} \\
\varepsilon_{22} \\
\varepsilon_{33} \\
2 \varepsilon_{12} \\
2 \varepsilon_{13} \\
2 \varepsilon_{23}
\end{array}\right\}=\left[\begin{array}{cccccc}
\frac{1}{E_{A}} & -\frac{\nu_{A}}{E_{A}} & -\frac{\nu_{A}}{E_{A}} & 0 & 0 & 0 \\
-\frac{\nu_{A}}{E_{A}} & \frac{1}{E_{T}} & -\frac{\nu_{T}}{E_{T}} & 0 & 0 & 0 \\
-\frac{\nu_{A}}{E_{A}} & -\frac{\nu_{T}}{E_{T}} & \frac{1}{E_{T}} & 0 & 0 & 0 \\
0 & 0 & 0 & \frac{1}{G_{A}} & 0 & 0 \\
0 & 0 & 0 & 0 & \frac{1}{G_{A}} & 0 \\
0 & 0 & 0 & 0 & 0 & \frac{2\left(1+\nu_{T}\right)}{E_{T}}
\end{array}\right]\left\{\begin{array}{l}
\sigma_{11} \\
\sigma_{22} \\
\sigma_{33} \\
\sigma_{12} \\
\sigma_{13} \\
\sigma_{23}
\end{array}\right\}
$$

Different homogenization methods are available in the literature to provide estimates of the elastic moduli, see Barbero et al. [15], Chen et al. [16], Jacques et al. [17]. One can cite the homogenization methods of Mori-Tanaka, the composite cylinder assemblage method, the self-consistent method, or numerical homogenization by finite element method. The analytical Mori-Tanaka scheme is well adapted for particulate composite but a large volume content of fibers can be detrimental for the prediction accuracy.

\subsubsection{Mori-Tanaka scheme for long fibers}

The Mori-Tanaka scheme for two-phase composite material with long fibers is well-established in the literature and can be found elsewhere. Weng [23], has shown an exact correspondence of the Mori-Tanaka model with the Hashin Strickman (lower) bound when the matrix is softer. The derivation of the model and related analytical expressions can be found for instance in Mura [24], Hill [25] or Hashin [26]. The homogenized yarn behavior is transversely isotropic when the phase behavior of the inclusions (long fibers) and of the matrix is elastic isotropic. We denote by $E_{m}, \nu_{m}$ (respectively $E_{i}, \nu_{i}$ ) the Young's modulus and Poisson's ratio of the matrix (respectively of the fibers). $f$ is the volume fraction of long fibers. 
Following notation of Eq. (8), the homogenized expressions for the transverse plain strain bulk modulus $k_{T}$ and shear modulus $G_{T}$ are found in Mura [24] :

$$
\begin{aligned}
k_{T} & =k_{m}+f\left(k_{i}-k_{m}\right) \frac{\left(k_{m}+\mu_{m}\right)}{\left(k_{m}+\mu_{m}\right)+(1-f)\left(k_{i}-k_{m}\right)} \\
G_{T} & =\mu_{m}+\frac{2 \mu_{m} f\left(\mu_{i}-\mu_{m}\right)\left(k_{m}+\mu_{m}\right)}{2 \mu_{m}\left(k_{m}+\mu_{m}\right)+(1-f)\left(\mu_{i}-\mu_{m}\right)\left(k_{m}+2 \mu_{m}\right)}
\end{aligned}
$$

where the plain strain bulk modulus $k_{i}$ of the inclusion (resp. $k_{m}$ of the matrix) is linked to the Young's modulus and Poisson's ratio of the inclusion (resp. of the matrix):

$$
k_{i}=\frac{E_{i}}{2\left(1-\nu_{i}-2 \nu_{i}^{2}\right)} \quad k_{m}=\frac{E_{m}}{2\left(1-\nu_{m}-2 \nu_{m}^{2}\right)}
$$

and $\mu_{i}$ and $\mu_{m}$ are the shear moduli of the inclusion and matrix. From Eqs. (9) and (10), the transverse Young's modulus $E_{T}$ and Poisson's ratio $\nu_{T}$ are obtained:

$$
\begin{gathered}
E_{T}=\frac{G_{T}\left(3 k_{T}-G_{T}\right)}{k_{T}} \\
\nu_{T}=\frac{-G_{T}+k_{T}}{2 k_{T}}
\end{gathered}
$$

The expression of the axial elastic properties $E_{A}, G_{A}$ and $\nu_{A}$ are taken from Hill [25] and Hashin [26]:

$$
\begin{gathered}
E_{A}=f E_{i}+E_{m}(1-f)+\frac{4\left(\nu_{i}-\nu_{m}\right)^{2} f(1-f)}{\frac{f}{k_{m}}+\frac{1-f}{k_{i}}+\frac{1}{\mu_{m}}} \\
G_{A}=\mu_{m} \frac{\mu_{i}(1+f)+\mu_{m}(1-f)}{\mu_{i}(1-f)+\mu_{m}(1+f)} \\
\nu_{A}=f \nu_{i}+\nu_{m}(1-f)+\frac{\left(\nu_{i}-\nu_{m}\right)\left(\frac{1}{k_{m}}-\frac{1}{k_{i}}\right) f(1-f)}{\frac{f}{k_{m}}+\frac{1-f}{k_{i}}+\frac{1}{\mu_{m}}}
\end{gathered}
$$

\subsubsection{Generation of the numerical unit cell}

Since the volume fraction of fibers is larger than sixty percent, we propose to compare the Mori-Tanaka homogenization method with numerical homogenization adopting 3D and 2D finite element models, based on periodic unit cell. In order to be as representative as possible of the studied laminate, the radii of fibers in the unit cell will be distributed according to the Burr distribution (Eq. (6), as measured in each yarn) and the volume fraction of fibers corresponds to the experimental one. A large number of instances will be also considered to capture the effect of the spatial distribution of fibers on elastic predictions; different positions of the fibers in the unit cell leading to different homogenized predictions.

It exists an important literature concerning the definition of representative volume element for composite materials, see for instance Torquato [28]. The method called random sequential

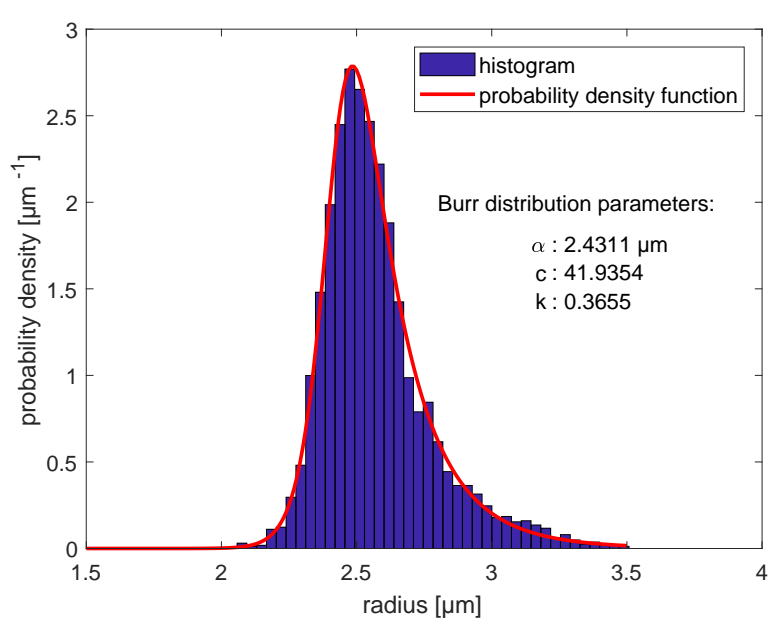

Figure 6: Probability density function of the fiber radius. The probability density function has been fitted by a Burr distrubtion, see Eq. (6). The parameters adopted for the Burr distribution are: $c=41.935, k=0.366$ and $\alpha=2.43 \mu \mathrm{m}$. The mean radius is $2.57 \mu \mathrm{m}$ with a standard deviation of $0.2 \mu \mathrm{m}$. The fiber radius is ranging from $2 \mu \mathrm{m}$ to $3.5 \mu \mathrm{m}$.

addition (RSA), as presented in the work of Smith [29], is frequently considered in the literature for the generation of particulate composite microstructure. For a $2 \mathrm{D}$ microstructure, the principle is to add sequentially disks. As a new disk is added, it may overlap with another disk in the unit cell. When such condition is met, then the last added disk is removed. Another position is chosen. The disk position is validated when it does not meet any already pre-existing disks. The main drawback of the method is the jamming limit which occurs for cylinders for a volume fraction of approximately $55 \%$. Since the volume fractions of fibers measured in our material are respectively $62 \%$ and $67 \%$ for warp and fill directions, the RSA scheme is not efficient for the present work. Therefore, a different strategy has been adopted based on the work of Lubachevsky [27]. The main steps are recalled in the following. First a set of disks (representing fibers with very small radius) is generated. At $t=0$, nuclei (in the sense that they have small radius) of disks are distributed randomly in the RVE and a random speed is assigned to each disk. Since the disks are very small, the probability that two disks overlap is low. As time increases, disks grow, the number of collision increases. When two disks (considered as rigid) get in contact, they bounce as billiard balls. To prevent vanishing distance between two fibers in the RVE, leading to very small or distorted elements, a small offset of $0.05 \mu \mathrm{m}$ is added to the contact criteria during the generation of the RVE so that fibers bounce when they are at this distance. When a disk crosses the remote boundary of the RVE, it is duplicated by periodicity on the opposite side. The computation stops when the total area of the disks reaches the requested value, i.e. when the targeted volume fraction of fibers is reached (Fig. 7). In our approach, fibers do not have a unique radius. A dedicated program is scaling the initial distribution of the fibers (at $t=0$ ) so that the final distribution of radii of the generated disks fits the measured Burr distribution (when the volume fraction of 


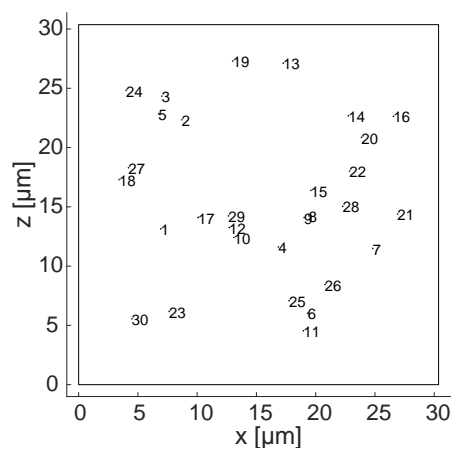

(a) Step 1

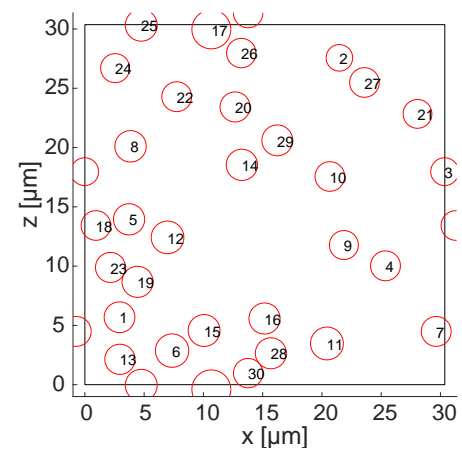

(b) Step 2

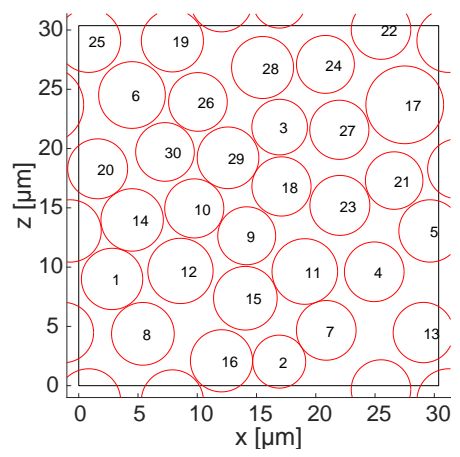

(c) Step 3

Figure 7: Generation of a 2D representative unit cell containing 30 fibers. The Lubachevsky method [27] is adopted, since the volume fraction of fibers is larger than the jamming limit of the random sequential addition scheme. (a) At time $t=0$, nuclei are randomly distributed and a random velocity is prescribed to all disks. Note that the radii are heterogeneous, following a Burr distribution. (b) As time increases, the radius of all disks grows homothetically. (c) The growth progress is stopped when the targeted volume fraction is met. More details can be found in Appendix A.

fibers is reached). This ensures a good description of the real structure. More details can be found in Appendix A.

At the end of the process, the center positions and radii of all fibers in the unit cell are transfered into Abaqus software. For that purpose, a dedicated Python script reads a file in which the positions and radii are listed and draws disks in the unit cell. One finally gets a 2D RVE of fibers in a matrix. To obtain a 3D $\mathrm{RVE}$, one only proceeds by extrusion along the third direction. In our case, the 3D RVE is a cube but the infinite length of the fibers is taken into account by adopting periodic boundary conditions.

In our work, a statistical analysis is developed. We do not restrict our analysis to a single configuration. Various sets have been generated, each of them leading to a new configuration, satisfying both the prescribed volume fractions of fibers and distribution of fiber radius.

Finite element calculations on the unit cell are carried out with periodic boundary conditions (PBC) prescribed at the remote boundary of the RVE. More details on PBCs are given in Appendix B. For the 3D model, six loading conditions are created: three tensions and three shears. For the 2D model (plane strain), only three loading conditions are applied: two tensions and one shear. For both 2D and 3D unit cell, a small displacement is imposed on one face of the RVE and the resulting homogenized strains and stresses are calculated by volume averaging of strains and stresses over all elements:

$$
X_{\text {moy }}=\sum_{e=1}^{n} \frac{X^{e} V^{e}}{\sum_{e=1}^{n} V^{e}}
$$

In our approach, $X$ stands for stress or strain components and $X^{e}$ the corresponding quantities at the element level. $V^{e}$ is the volume of the element and $n$ the total number of elements in the model.

\subsubsection{Selection of a salient unit cell}

Figs. $8 \mathrm{a}$ and $8 \mathrm{~b}$ present the unit cells generated by the Lubachevsky [27] scheme, as presented in the previous section 3.2.2. The $3 \mathrm{D}$ model is obtained from the $2 \mathrm{D}$ unit cell by a simple extrusion along the fiber direction, so the number, radii and positions of fibers are identical. The radius of the fibers is varying according to the Burr probability density function, relationship (6). In order to evaluate the effect of the radius heterogeneity in the unit cell on the elastic predictions, monodisperse 2D RVE have been generated with still random position of fibers but considering that all fibers have the same radius, see Fig. 8c. A unit cell with face centered cubic distribution of fibers is also proposed, see Fig. 8d. The cells presented in Figs $8 \mathrm{a}$ to $8 \mathrm{c}$ contain thirty fibers. In the last part of this section, the number of fibers in the unit cell will be varied. The fibers are aligned with the direction 1 and the volume fraction of fibers is set to $67 \%$ as a reference (corresponding to the fill yarn). Note that a mesh convergence study has been carried out for all FEM models. It has been checked that the overall elastic moduli are mesh insensitive for the mesh density with approximately 8000 elements of type CPE3 and CPE4R (plane strain elements, triangles and quadrangles) for a 2D cell containing 30 fibers.

For purpose of comparison, the elastic behavior of fibers and matrix is considered as linear isotropic with the following set of elastic moduli:

- glass fibers: $E_{i}=72.3 \mathrm{GPa}, \nu_{i}=0.22$

- matrix: $E_{m}=4.73 \mathrm{GPa}, \nu_{m}=0.167$

Note that the considered values for the matrix are consistent with future results of Section 3.4.

The predictions based on the 3D RVE (Fig. 8a) are considered as the reference and are compared in Table 3 to those obtained based on unit cells presented in Figs $8 \mathrm{~b}$ to $8 \mathrm{~d}$. For all unit cells considered in the present paper, it has been checked that $E_{2}=E_{3}, \nu_{12}=\nu_{13}$ and $G_{12}=G_{13}$. It has also been checked that $G_{23}$, obtained by prescribing a shear loading to the RVE, satisfies $G_{23}=E_{2} /\left(2\left(1+\nu_{23}\right)\right)$ so the random generation of fibers in the unit cell provides a transverse isotropic response. Note that the estimates provided by the Mori-Tanaka scheme is also included in this table.

From Table 3, it is observed that predictions based on unit cells displayed in Figs $8 \mathrm{~b}$ and $8 \mathrm{c}$ provide similar results. It 


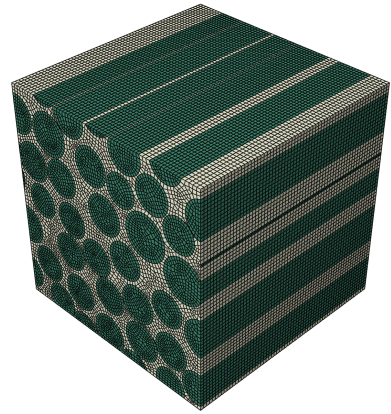

(a)

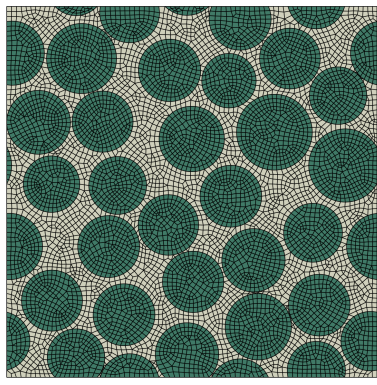

(b)

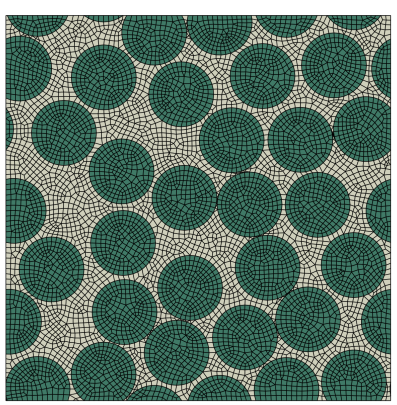

(c)

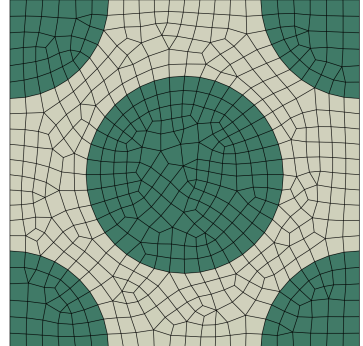

(d)

Figure 8: Presentation of the four configurations used for the yarn homogenization. In each model, fiber volume fraction of $67 \%$ is considered (representative of the fill yarn). (a) Presentation of the 3D model. The fiber radii distribution has been determined based on SEM images and described by a Burr probability density function (6), (b) the corresponding 2D model with exactly the same position for all fibers. Radii are still heterogeneous. (c) 2D VER with a unique fiber radius. (d) simple unit cell model with fibers placed on a regular grid (face centered symmetry).

$67 \%$ fiber volume fraction

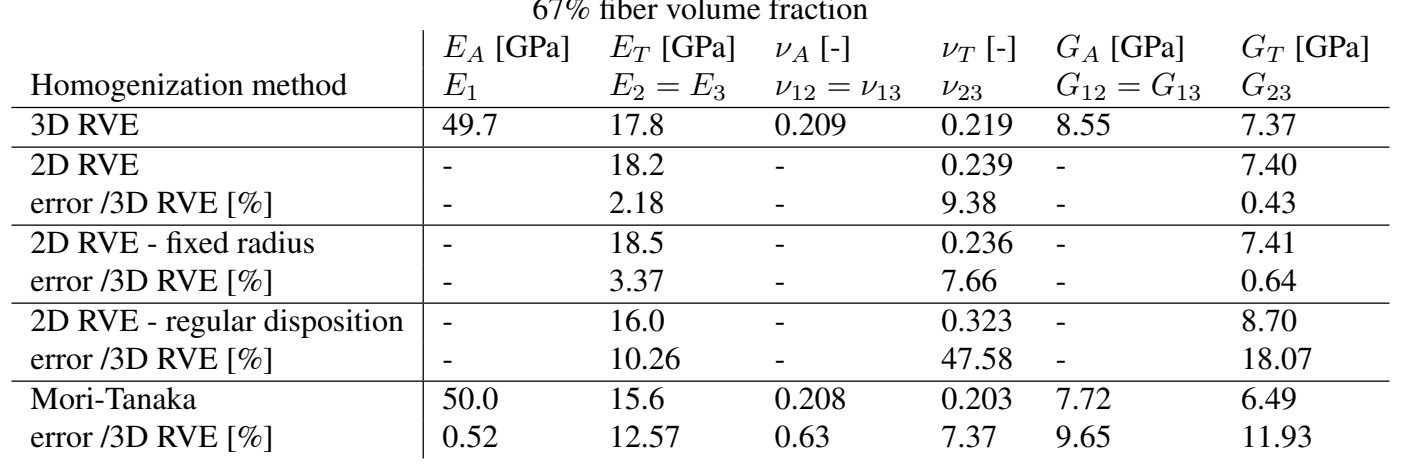

Table 3: Predictions of elastic properties at the level of the yarn. The yarn elastic response is transverse isotropic as presented in Eq. (8). Comparison between four different numerical models based on a 3D RVE, a 2D RVE, a 2D RVE where fibers have the same radius, a 2D RVE with a face centered cubic symmetry. Mori-Tanaka homogenization results are also presented. Fibers are aligned with direction 1 . A fiber volume fraction of $67 \%$ is considered.

seems that for the case considered here, taking into account the radii distribution leads to slight changes in the predictions. This is probably due to the small heterogeneity of the fiber radii, illustrated by a standard deviation of $0.2 \mu \mathrm{m}$ and a mean radius of $2.57 \mu \mathrm{m}$. The 2D model (Fig. 8d) with a face centered cubic distribution of fibers is not able to provide accurate estimates for a composite with randomly distributed fibers. For instance, a $48 \%$ error is observed when considering the value of the Poisson's ratio $\nu_{23}$ when compared to the 3D RVE. This unit cell will be avoided for our study.

The determination of the transverse elastic properties $\left(E_{2}\right.$, $\left.\nu_{23}, G_{23}\right)$ requires $2 \mathrm{D}$ or $3 \mathrm{D}$ unit cells. It is observed that the $3 \mathrm{D}$ simulation and the corresponding $2 \mathrm{D}$ plane strain simulation provide close results. Nevertheless $2 \mathrm{D}$ simulations are less time consuming than $3 \mathrm{D}$ computations. It is also observed that the Mori-Tanaka homogenization scheme gives accurate results for the prediction of the elastic properties relative to the direction of fibers $\left(E_{1}, \nu_{12}=\nu_{13}\right.$ and $\left.G_{12}=G_{13}\right)$ and less accurate estimates for the transverse elastic moduli $\left(E_{2}=E_{3}, \nu_{23}\right.$ and $G_{23}$ ). As a consequence, a hybrid model is adopted in the following to compute the homogenized properties of a yarn. The elastic properties relative to the axial direction are computed with Mori-Tanaka scheme and the transverse properties with the $2 \mathrm{D}$ model corresponding to the unit cell of Fig. $8 \mathrm{~b}$.

Results of Table 3 are obtained for a fixed configuration. From SEM observations, it has been noted that the spatial distribution of fibers may change from yarns to yarns. So to investigate this spatial distribution effect, a large number of 2D RVE has been considered. Predictions are compared to evaluate the influence of different seeds of fibers. Of course, each configuration is based on the same radii distribution as measured by SEM (see section 1), contains the same number of fibers and the same volume content of fibers. Hundred random configurations have been generated and simulated. For each configuration, the homogenized elastic properties $\left(E_{2}, \nu_{23}, G_{23}\right)$ have been computed. Fig. 9 displays the predicted heterogeneity (based on histogram) generated by hundred configurations containing 30 fibers with a fiber volume fraction of $67 \%$. The vertical line represents the average value. One can see that properties vary from one configuration to another. Considering only one single configuration can lead to a result quite far from the average value (more than ten percent difference). Next, for the homogenization of the woven composite, the configuration providing the closest predictions from the average for the three quantities 
$\left(E_{2}, \nu_{23}, G_{23}\right)$ will be selected. It should be noted that results shown in Table 3 are deduced based on this configuration.

The effect of the number of inclusions in a RVE has been tested by considering various sizes of unit cell. In the present work, we have adopted RVE containing 10, 30, 50 and 70 fibers respectively. A hundred RVE (corresponding to Fig. 8b) has been generated for each case (400 simulations have been conducted in total). The volume fraction of fibers is $67 \%$ and the fiber radius is following the Burr distribution (Eq. (6)) in each considered case. Fig. 10 presents the average value and the standard deviation of the predicted homogenized transverse Young's modulus when the number of fibers is increased from 10 to 70 . It can be noticed that the mean value (based on hundred configurations) is constant regardless the number of fibers in the RVE. However the standard deviation decreases when the unit cell contains a larger number of fibers. Since only the configuration leading to the closest predictions (with respect to the average values) is selected for the homogenization of the yarn and since the average value of the transverse Young's modulus does not depend on the number of fibers (see Fig. 10), a configuration with 30 fibers is selected, leading to reasonable computation times.
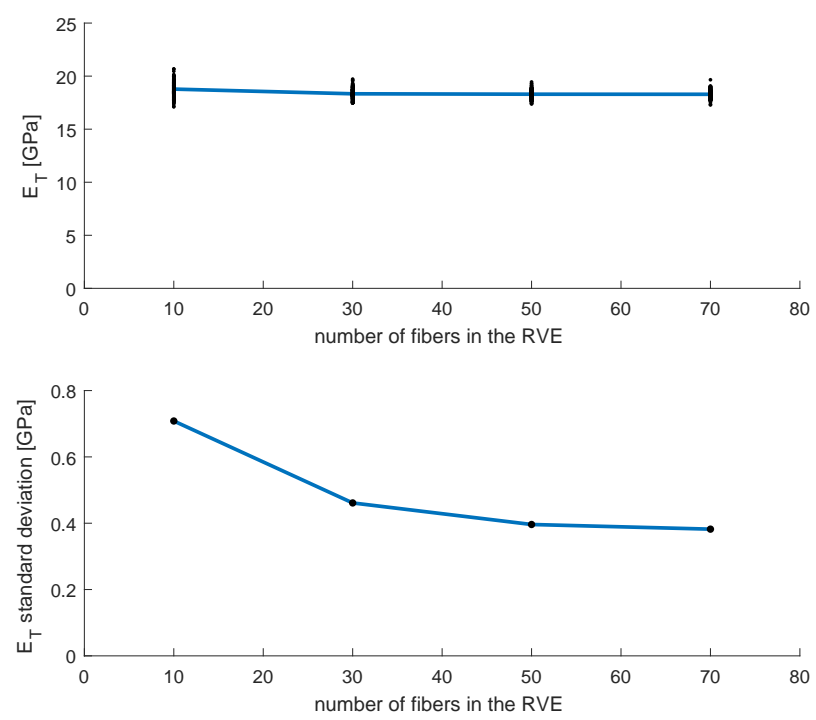

Figure 10: Effect of the number of fibers in a 2D RVE on (a) the average transverse Young's modulus $E_{T}$, (b) the associated standard deviation. The volume fraction of fibers is fixed and equal to $67 \%$. The prediction of $E_{T}$ for each configuration is plotted in (a) with a black dot. The solid line shows the evolution of the average value. It is observed that as the number of fibers is increased, the predictions are more homogeneous leading to the decrease of the standard deviation. Note that as concerned the overall transverse Young's modulus, the average value is not evolving with the number of fibers.

The same computations have also been carried out for lower fiber volume fractions: $50 \%$ and $30 \%$ (see Tables 4 and 5). As expected, it is observed that the difference between predictions based on a random distribution of fibers and on one hand the cubic unit cell or on the other hand the Mori-Tanaka approach decreases when the fiber volume fraction is lower. Neverthe-
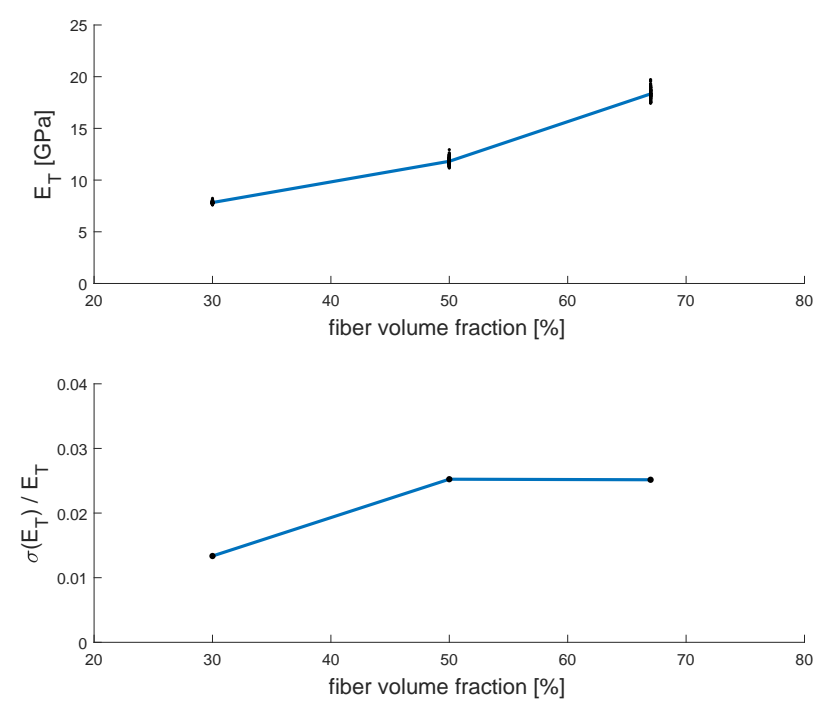

Figure 11: Effect of the volume fraction of fibers in a 2D RVE on (a) the average transverse Young's modulus $E_{T}$, (b) the associated relative standard deviation $\sigma\left(E_{T}\right) / E_{T}$. Each configuration contains 30 fibers. The value of $E_{T}$ for each configuration is plotted with a black dot in (a). The solid line depicts the evolution of the average value when the volume fraction of fibers increases. As expected for low volume fraction of fibers, the discrepency between various instances is quite small. The relative standard deviation is increased when the volume fraction of fibers increases from $30 \%$ to $67 \%$.

less, even for $30 \%$ fiber content, a random fiber distribution in the unit cell is still compulsory to describe properly the yarn behavior. Fig. 11 presents the evolution of the transverse Young's modulus $E_{T}$ for the three volume fractions considered in this paper $(30 \%, 50 \%$ and $67 \%)$. Thirty fibers are present in the unit cell. Numerous configurations have been tested for each case, so that the deviation between instances can be estimated. As expected, as the volume content of fibers increases, the heterogeneity in predictions increases. The relative standard deviation, defined as the ratio between the standard deviation and the mean value, is growing significantly when the volume fraction increases from $30 \%$ to $50 \%$. Therefore, it is important to consider a larger number of configurations for large volume content of fibers, so that the average value of the elastic properties is statistically obtained.

\subsection{Homogenization of the woven composite}

The laminate geometry has been defined precisely based on the measurements carried out by microtomography and SEM, as seen in section 2.2. The numerical model is built on Abaqus. The main steps for the RVE definition have been presented previously. Fig. 12 presents the adopted geometry for the numerical calculations where part of the matrix phase has been hidden to precisely show the yarns and their geometry.

The transverse isotropic behavior of the yarns is computed with the method described in section 3.2.3 while the matrix surrounding the yarns is considered as elastic isotropic. Due to the weaving pattern, a local frame of anisotropy is attached to 


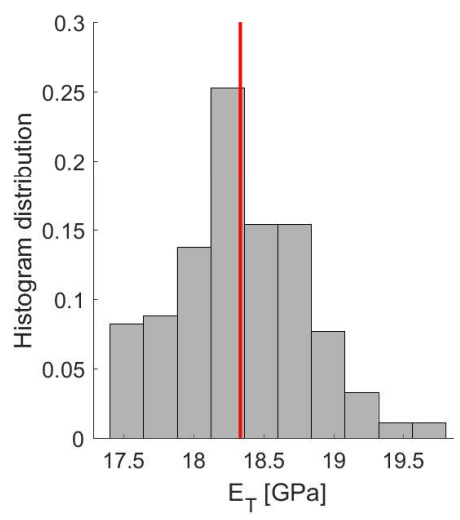

(a)

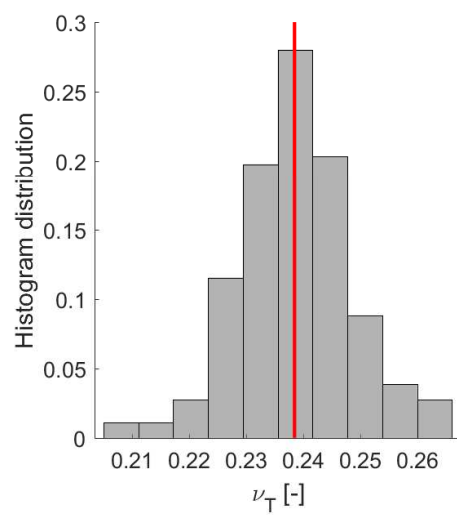

(b)

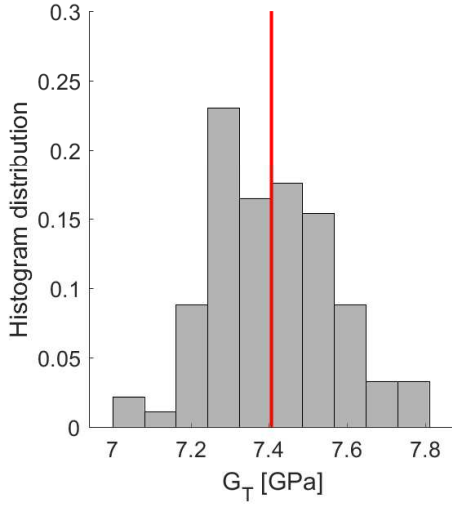

(c)

Figure 9: Prediction of the transverse elastic properties of the yarn, based on hundred RVE with 30 fibers. A fiber volume fraction of $67 \%$ is considered. Results are obtained by considering $2 \mathrm{D}$ unit cell (see Fig. 8b). a) Transverse Young modulus $E_{T}$. b) Transverse Poisson ratio $\nu_{T}$. c) Transverse shear modulus $G_{T}$. It has been checked for all configurations that $E_{2}=E_{3}=E_{T}, \nu_{23}=\nu_{32}=\nu_{T}$. The present predictions are representative of the fill yarn behavior. For the present finite element model, glass fiber and matrix properties are: $E_{i}=72.3 \mathrm{GPa}, \nu_{i}=0.22, E_{m}=4.73 \mathrm{GPa}, \nu_{m}=0.167$.

$50 \%$ fiber volume fraction

\begin{tabular}{l|llllll} 
& $E_{A}[\mathrm{GPa}]$ & $E_{T}[\mathrm{GPa}]$ & $\nu_{A}[-]$ & $\nu_{T}[-]$ & $G_{A}[\mathrm{GPa}]$ & $G_{T}[\mathrm{GPa}]$ \\
Homogenization method & $E_{1}$ & $E_{2}=E_{3}$ & $\nu_{12}=\nu_{13}$ & $\nu_{23}$ & $G_{12}=G_{13}$ & $G_{23}$ \\
\hline 3D RVE & 38.2 & 11.6 & 0.201 & 0.217 & 5.51 & 4.72 \\
\hline 2D RVE & - & 11.8 & - & 0.232 & - & 4.76 \\
error /3D RVE [\%] & - & 2.14 & - & 6.80 & - & 0.84 \\
\hline 2D RVE - regular disposition & - & 10.3 & - & 0.293 & - & 5.22 \\
error /3D RVE [\%] & - & 10.61 & - & 34.75 & - & 10.62 \\
\hline Mori-Tanaka & 38.5 & 10.6 & 0.200 & 0.195 & 5.16 & 4.42 \\
error /3D RVE [\%] & 0.91 & 8.65 & 0.55 & 9.98 & 6.31 & 6.33
\end{tabular}

Table 4: Predictions of elastic properties at the level of the yarn. The yarn elastic response is transverse isotropic as presented in Eq. (8). Comparison between four different numerical models based on a 3D RVE, a 2D RVE, a 2D RVE where fibers have the same radius, a $2 \mathrm{D}$ RVE with a face centered cubic symmetry. Mori-Tanaka homogenization results are also presented. Fibers are aligned with direction 1 . A fiber volume fraction of $50 \%$ is considered

\begin{tabular}{l|llllll}
\multicolumn{7}{c}{$30 \%$ fiber volume fraction } \\
Homogenization method & $E_{A}[\mathrm{GPa}]$ & $E_{T}[\mathrm{GPa}]$ & $\nu_{A}[-]$ & $\nu_{T}[-]$ & $G_{A}[\mathrm{GPa}]$ & $G_{T}[\mathrm{GPa}]$ \\
\hline 3D RVE & $E_{1}$ & $E_{2}=E_{3}$ & $\nu_{12}=\nu_{13}$ & $\nu_{23}$ & $G_{12}=G_{13}$ & $G_{23}$ \\
\hline 2D RVE & 24.8 & 7.70 & 0.189 & 0.211 & 3.55 & 3.17 \\
error /3D RVE [\%] & - & 7.82 & - & 0.225 & - & 3.18 \\
\hline 2D RVE - regular disposition & - & 1.61 & - & 6.65 & - & 0.49 \\
error /3D RVE [\%] & - & 7.39 & - & 0.251 & - & 3.31 \\
\hline Mori-Tanaka & - & 4.09 & - & 19.34 & - & 4.68 \\
error /3D RVE [\%] & 25.0 & 7.37 & 0.189 & 0.186 & 3.46 & 3.11 \\
& 0.94 & 4.35 & 0.30 & 11.86 & 2.53 & 1.89
\end{tabular}

Table 5: Predictions of elastic properties at the level of the yarn. The yarn elastic response is transverse isotropic as presented in Eq. (8). Comparison between four different numerical models based on a 3D RVE, a 2D RVE, a 2D RVE where fibers have the same radius, a 2D RVE with a face centered cubic symmetry. Mori-Tanaka homogenization results are also presented. Fibers are aligned with direction 1 . A fiber volume fraction of $30 \%$ is considered

each yarn. The transverse isotropy axis is tangent to the central axis of the yarn which follows a cosine curve. However, it has been observed by simulations that the yarn oscillation being small, considering the local frame induces a difference on homogenized values as small as $1.4 \%$. This is observed for all estimated elastic moduli.

The RVE represents the elementary cell of the woven composite. The whole composite can be generated by translating the RVE of any vector $k \cdot\left(2 b_{w}, 2 b_{f}, 0\right)$ ( $k$ being an integer). It is important to mention that the RVE has the thickness of the composite so the periodicity of the RVE does not exist in direction 3. Loadings are prescribed to the RVE by adopting Periodic Boundary Conditions (PBC). They are implemented as described in the work of Pierard et al. [30], at least for the in-plane loading, see Appendix B.

Three tensile and three shear loadings are prescribed inde- 


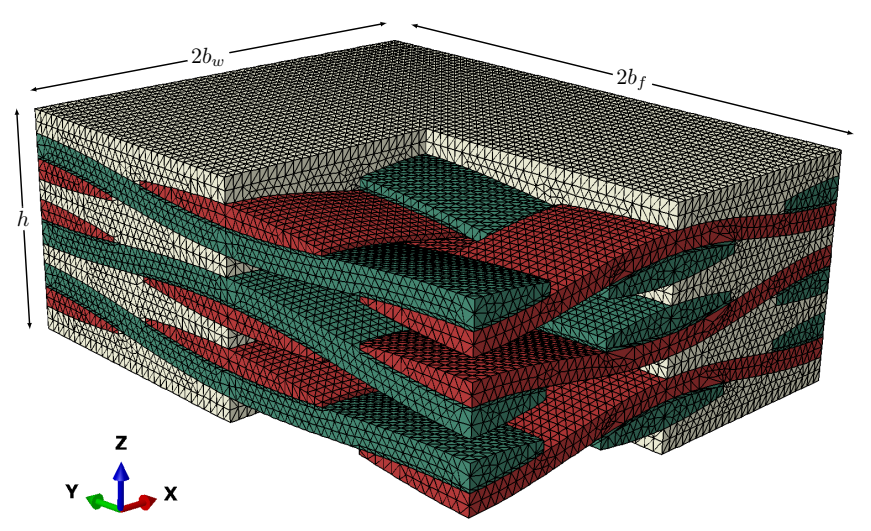

Figure 12: 3D representative volume element. The laminate is made of three layers of intertwinned warp and fill yarns. The size of the RVE is $2 b_{w}$ in the warp direction, $2 b_{f}$ in the fill direction. The thickness of the laminate is $h$. The model will be adopted for the derivation of the orthotropic behavior of the laminate. Note that since the matrix phase is unknown, an inverse method is compulsory. The model contains around 360000 tetrahedral elements. A mesh convergence study has been carried out to check that the proposed mesh is dense enough to provide mesh insensitive predictions (at least for the overall elastic properties).

pendently on the RVE to get the overall orthotropic elastic behavior as presented in Eq. (1). Indeed, from the numerical calculations, average strains $\varepsilon^{a v g}$ and stresses $\sigma^{a v g}$ are computed by a summation over all elements of the RVE, see Eq. (17). From the simulations of the three uniaxial tensions along direction $\bar{e}_{i}$, the homogenized moduli $E_{i}$ and $\nu_{i j}$ can be computed (no summation on the indices, $j$ refers to $\bar{e}_{j}$ direction normal to $\left.\bar{e}_{i}\right)$ :

$$
\begin{gathered}
E_{i}^{s i m}=\frac{\sigma_{i i}^{a v g}}{\varepsilon_{i i}^{a v g}} \\
\nu_{i j}^{s i m}=-\frac{\varepsilon_{j j}^{a v g}}{\varepsilon_{i i}^{a v g}}
\end{gathered}
$$

From the three shear loadings, the effective shear moduli are obtained:

$$
G_{i j}^{s i m}=\frac{\sigma_{i j}^{a v g}}{2 \varepsilon_{i j}^{a v g}}
$$

The numerical homogenization computations have been performed with two mesh densities in order to verify that results are mesh insensitive. The two models contain respectively 360,000 and 597,000 tetrahedral elements C3D10 (with quadratic interpolation). It has been checked that the two models give the same homogenized values.

This numerical homogenization method works when the material behavior of each phase is known.

\subsection{Optimization, inverse identification}

In our study, the behavior of the glass fiber is supposed known $\left(E_{f}=72.3 \mathrm{GPa}, \nu_{f}=0.22\right.$, see Chen et al. [16] and Barbero et al. [15]). But the resin present in the investigated laminate is not clearly described by the provider and its behavior is unknown. In fact, the resin is a thermoset. So it is really difficult to extract the resin behavior directly from the laminate. In addition, in contrast to what is usually reported in the literature, the matrix phase is not a pure polymeric resin. Indeed, for high frequency applications, as observed on SEM, the matrix surrounding the yarns is filled with inclusions, whereas the matrix inside the yarns contains no inclusions (see Fig. 4). So in addition to the glass fiber, the laminate is made of two phases (intra-strand resin and inter-strand matrix), whose elastic behaviors are assumed isotropic but unknown. Their elastic responses will be identified by an inverse method, by comparing the predictions of the 3D model with the measured in-plane global elastic moduli of the laminate $\left(E_{1}^{\exp }, E_{2}^{\exp }, \nu_{12}^{\exp }, G_{12}^{\text {exp }}\right)$.

Four unknown parameters are introduced: $E_{m}, \nu_{m}$ (respectively $\left.E_{m t}, \nu_{m t}\right)$ the Young's modulus and Poisson's ratio of the inter-strand matrix (respectively intra-strand resin). The inverse method is an iterative process, as illustrated on Fig. 13. The reliability of the prediction is measured by a cost function, defined as the sum of the squared differences between simulation and experiment:

$$
\begin{aligned}
f_{\text {cost }}= & \left(\frac{E_{1}^{\text {exp }}-E_{1}^{\text {sim }}}{E_{1}^{\exp }}\right)^{2}+\left(\frac{E_{2}^{\text {exp }}-E_{2}^{s i m}}{E_{2}^{\text {exp }}}\right)^{2} \\
& +\left(\frac{\nu_{12}^{\text {exp }}-\nu_{12}^{s i m}}{\nu_{12}^{\text {exp }}}\right)^{2}+\left(\frac{G_{12}^{\text {exp }}-G_{12}^{s i m}}{G_{12}^{\text {exp }}}\right)^{2}
\end{aligned}
$$

The set of parameters $\left(E_{m}, \nu_{m}, E_{m t}, \nu_{m t}\right)$ is obtained by minimizing the cost function $f_{\text {cost }}$ using a Nelder-Mead algorithm coupled with Abaqus by a dedicated Python script. First, an initial set for the four unknown parameters $\left(E_{m}^{i}, \nu_{m}^{i}, E_{m t}^{i}\right.$, $\left.\nu_{m t}^{i}\right)$ is defined. Based on the initial trial set, a homogenization process is performed on a $2 \mathrm{D}$ numerical RVE to estimate the transverse isotropic behavior of the warp and fill yarns, the Mori-Tanaka homogenization being used for the remaining elastic moduli. Computations are repeated since the two yarns have a different fiber volume fraction. The computed elastic transverse isotropic behaviors of the fill and warp yarns are introduced in the 3D homogenization model. Three simulations are carried out: tension along $\overline{e_{1}}$ axis, tension along $\overline{e_{2}}$ axis and one in-plane shear. As a result, four homogenized elastic moduli $E_{1}^{\text {sim }}, E_{2}^{\text {sim }}, \nu_{12}^{\text {sim }}$ and $G_{12}^{\text {sim }}$ are derived and compared to the in-plane measured data. By evaluating the cost function and using the Nelder-Mead minimization algorithm, new estimates $E_{m}, \nu_{m}, E_{m t}, \nu_{m t}$ are available. The simulations are performed once again with these new estimates.

For the case considered in the present work, the cost function decreases rapidly and saturates toward a limit value $f_{\text {cost }}^{\text {lim }}$ after some tenth of iterations. In the iterative optimization process, the calculations are stopped when an asymptotic value is reached, meaning that the value of $f_{\text {cost }}$ has not changed by more than $10^{-5}$ during 20 consecutive iterations. Nevertheless, in order to ensure that the solution is stable, the optimization procedure, based on the Nelder-Mead algorithm, is performed again with the previously calculated optimized values as initial guess. It has been checked that the limit value $f_{\text {cost }}^{\text {lim }}$ and the estimated parameters $E_{m}, \nu_{m}, E_{m t}, \nu_{m t}$ are unchanged. Once the convergence criterion is reached, estimates for the elastic behavior of the two unknown phases as well as of the fill and 


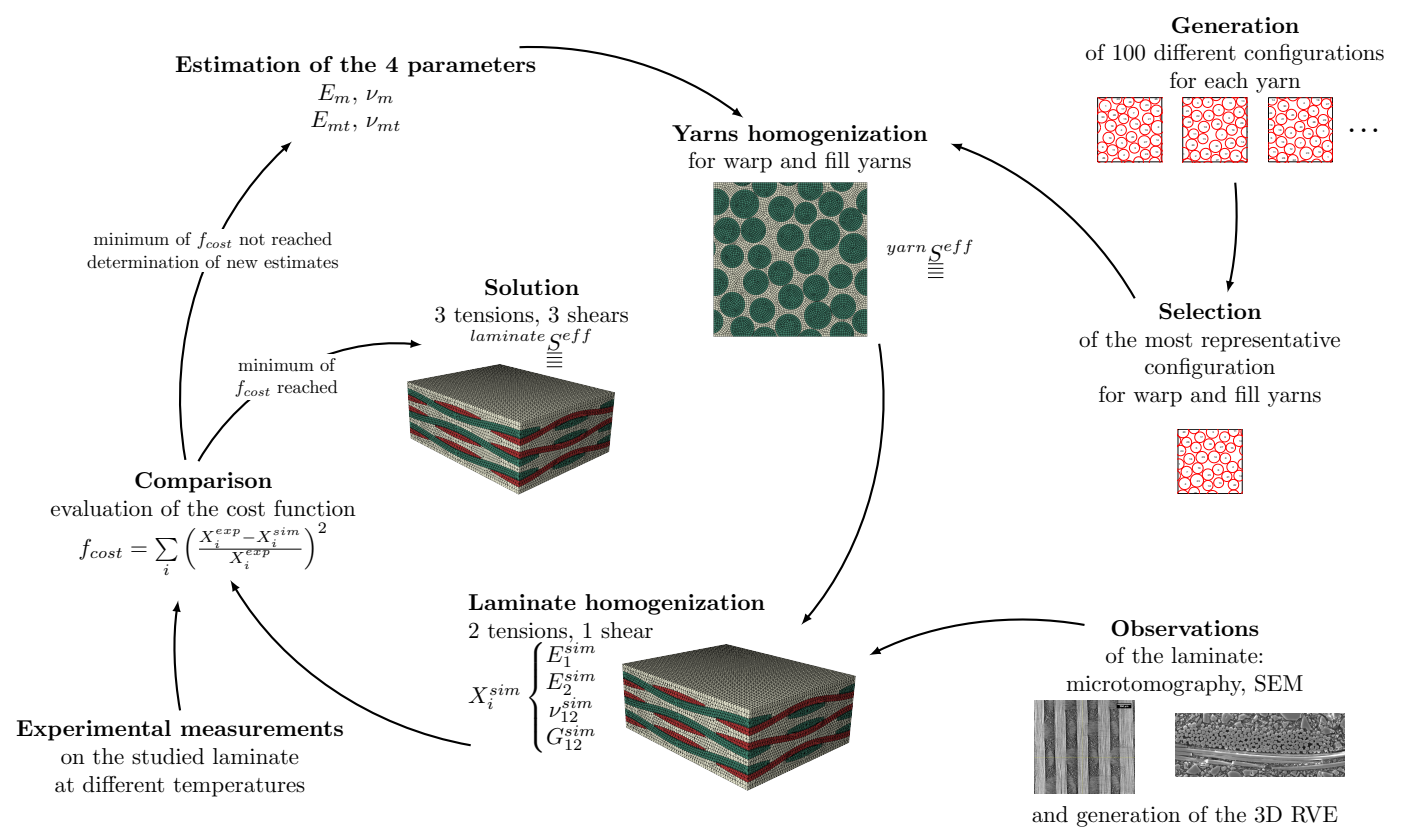

Figure 13: Global scheme for the inverse method. All the steps necessary to homogenize a woven composite are represented. An estimate of the behaviors of the two phases (intra-strand resin and inter-strand matrix) is given. A first homogenization is done at the yarn level with Mori-Tanaka model and a 2D RVE studied with Abaqus. The yarn is made of glass fibers and an intra-strand resin. This 2D RVE is selected from 100 random configurations to be the most representative. This is done for both warp and fill yarns. Next the RVE for the complete woven composite homogenization is generated from microtomography and SEM observations. The yarn and the inter-strand phase behaviors are defined or estimated. Three loadings are applied to the RVE so as to compute the four in-plane elastic moduli. In the next step, these four estimated elastic moduli are compared with the measured ones. A cost function is minimized with the use of a Nelder-Mead algorithm. New estimates are used and the whole homogenization cycle is performed again. When a convergence criterion is reached on the cost function, six loadings are prescribed to the 3D RVE to compute the orthotropic elastic behavior. The method is carried out at different temperatures.

warp yarns are obtained. Subsequently, the elastic orthotropic behavior of the laminate is predicted by considering the six unitary loadings (three uniaxial tensions and three shear loadings).

Different initial sets have been tested. The inter-strand matrix is filled with ceramic inclusions so its corresponding initial Young's modulus $E_{m}^{i}$ is chosen larger than the one of the intrastrand resin: $E_{m t}^{i}<E_{m}^{i}$. With this additional condition, the solution of the optimization process is insensitive to the initial guess. In our approach, the initial elastic properties of the intrastrand resin are taken from the work of Chen et al. [16] and are representative of an epoxy resin: $E_{m t}^{i}=3450 \mathrm{MPa}$ and $\nu_{m t}^{i}=0.37$. The initial guess for the inter-strand matrix is taken as $E_{m}^{i}=2 E_{m t}^{i}$ and $\nu_{m}^{i}=\nu_{m t}^{i}$

Experimentally, the elastic moduli have been measured at different temperatures in the range $\left[-50^{\circ} \mathrm{C} ;+125^{\circ} \mathrm{C}\right]$. Thus, this numerical strategy has been successively applied at different temperatures. It is observed in Fig. 14 that the elastic moduli are quite temperature insensitive in the range $\left[-50^{\circ} \mathrm{C} ;+20^{\circ} \mathrm{C}\right]$ but as the temperature overcomes room temperature, the decrease of the Young's modulus or of the shear modulus (not presented here) is really important. In addition, it must be emphasized that in the range $\left[-50^{\circ} \mathrm{C} ;+125^{\circ} \mathrm{C}\right]$, the value of the Young's modulus $E_{1}$ is reduced by a factor of two, see Fig. 14 . It is also observed that the value of the datasheet, which corresponds to the behavior of the woven composite characterized at room temperature and with a different glass fiber fabric, is overestimating the measured properties. Fig. 14 presents also the temperature evolution of the out-of-plane Young's modulus $E_{3}$. Due to the thickness of the laminate, such data are difficult to obtain by tests. It is also found in Fig. 14 that the value of Young's modulus $E_{3}$ is divided by a factor of three when the temperature is varied from $-50^{\circ} \mathrm{C}$ to $+125^{\circ} \mathrm{C}$.

\section{Conclusion}

The PCB industry is facing rapid evolution with a huge competition for developing high performing PCB. In space and military applications, achieving reliability of high performance PCB is a challenge as it is a complex construction made out of many different materials. Consequently, the reliability of the board cannot be assessed without a precise material description. Numerical simulations based only on the properties of the datasheet will generate predictions with a low degree of reliability. This can be problematic, especially when the goal is to predict the fatigue life of printed circuit boards during thermal cycles. With the proposed combined and multi-stage homogenization procedure, the temperature dependent behavior of the woven composite can be accurately described, even when the matrix phase has a complex internal structure and is unknown. Such deep and complete information are not usually available in the literature.

A deep investigation of the elastic behavior of the woven composite is proposed. It relies on measurement of the inplane elastic properties. To estimate out of plane properties, 


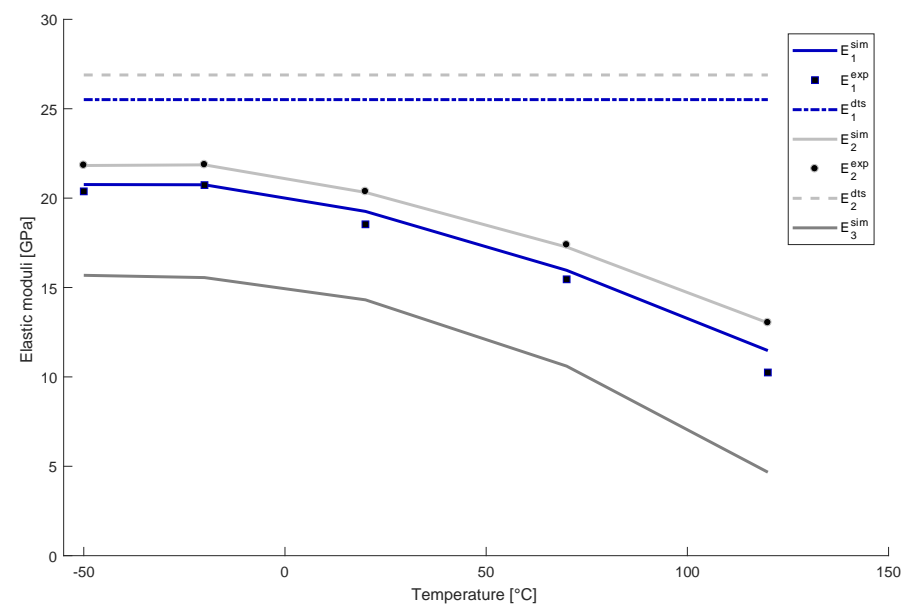

Figure 14: Predictions of the elastic moduli for the laminate. The in-plane elastic moduli $E_{1}$ and $E_{2}$ and the out of plane Young's modulus $E_{3}$ are seen to varying strongly with temperature. The prediction of the Young's modulus $E_{3}$ is the main outcome of the proposed work since the precise experimental determination of $E_{3}$ is difficult due to the small thickness of the laminate. Comparison with datasheet values proves the necessity of a careful characterization (experiments + numerical simulations) of the considered laminate. The proposed strategy has been employed in the temperature range $\left[-55^{\circ} \mathrm{C} ;+125^{\circ} \mathrm{C}\right]$. Thermal cycles in this range of temperature is a requirement of space standards during the qualification process of any printed circuit board.

the internal structure of the composite is quantified, based on micro-tomography scans and scanning electron microscope images. A new methodology is proposed to describe precisely the shape of the yarns. Owing to the large volume fraction of fibers in the yarns, 2D representative volume elements are generated by a scheme similar to the one proposed by Lubachevsky [27]. From observations, it has been checked that some variability in the fiber radius and in position of fibers exists from one yarn to another. As a consequence, a large number of configurations has been tested. The laminate considered in the present paper is designed for high frequency applications. The matrix is made of polymeric resin and filled with a large volume content of ceramic inclusions. The matrix behavior is not available, so an inverse method is proposed. Finally, 3D simulations at the level of the RVE enable to define the orthotropic behavior of the laminate. Therefore, as the main outcome of the work, the out of plane properties are evaluated. There is usually a lack of reliable data on these properties in the literature.

The considered laminate is part of a multi-layer PCB for space applications. During qualification process and operation, the PCB will face severe thermal cycles. For that purpose, the behavior of the laminate has been identified in the temperature range of interest for the applications, i.e. $\left[-50^{\circ} \mathrm{C} ;+125^{\circ} \mathrm{C}\right]$.

The proposed method is general enough and can be applied to other complex laminates and is not restricted to the evaluation of the thermo-elastic behavior. In the present work, we have enlightened how a combination of experimental set-ups and numerical simulations can provide precise predictions of the constitutive behavior of plain woven composite, dedicated to non-conventional structural applications like high frequency PCB.

\section{Acknowledgments}

The authors thank the support of CNES by means of the $\mathrm{PhD}$ grant of $\mathrm{G}$. Girard. The authors from LEM3 and CIMULEC thank the support of ANR through the program Labcom ANR14-LAB7-0003-01 for the creation of the labcom LEMCI. 


\section{Appendix A. 2D unit cell generation for composite with long fibers}

The procedure for the generation of multiple instances of 2D microstructure with inclusions (disks) of heterogeneous radius and for a given inclusion volume fraction $f$ is explained in this Section. The number of fibers $N$ in the unit cell is first chosen. In our approach, $N$ is varied in the range [10:70]. For each value of $N$, hundred configurations are tested. The $J$ th configuration is built as follows. From the Burr distribution (6), a set $S(J)$ of $N$ values for the radius is selected (a Matlab procedure has been used for that purpose). Let's denote $R(i, J)$ the radius of the $i^{\text {th }}$ disk in the configuration $J$. The total area of the $N$ disks is computed. Since the volume fraction of fibers is known ( $62 \%$ for the warp yarn and $67 \%$ for the fill yarn), the length $l(J)$ of the squared unit cell is determined:

$$
l(J)=\sqrt{\frac{\sum_{i=1}^{N} \pi R(i, J)^{2}}{f}}
$$

Among all configurations (one hundred for each set of parameters), it has been checked that $l(J)$ is changing only slightly.

Consider that at a given time $t$, the radius of the disks in the unit cell is $r_{i}(J, t)$ for the configuration $J$. To start the Lubachevsky [27] type of approach, the radius of all disks is downsized: $r_{i}(J, t=0)=R(i, J) / A$. $A$ is an arbitrary real number, set to $A=100$ in the present work. During the process, all disks grow homothetically:

$$
r_{i}(J, t)=r_{i}(J, t=0) \cdot \frac{t}{t_{0}} \cdot A
$$

where $t_{0}$ is an arbitrary time (here $t_{0}=1 \mathrm{~s}$ ). Since a random velocity is prescribed to all disks at time $t=0$ and their radius is small (the initial volume fraction of fibers being $0.006 \%$ ), they move along a straight line with a probability of collision relatively low. As they reach the border of the RVE, they are duplicated on the opposite side, to preserve periodicity. As disks begin to grow, the frequency of collisions increases. Note that during the process, disks are considered as rigid so they bounce like billiard balls. Note that an offset distance $\delta$ is introduced so that the collision between two disks is triggered when the distance between them is equal to this offset distance. $\delta$ has been introduced to avoid the situation where two disks are finally in contact. Indeed, from the numerical point of view, this offset distance eases to avoid too small and distorted elements. As the cell is meshed in Abaqus, this may induce convergence problem. At time $t=t_{0}$, the simulation is stopped. All disks have reached their final radius $r_{i}\left(J, t=t_{0}\right)=R(i, J)$, and the volume fraction of fibers is naturally $f$.

\section{Appendix B. Periodic boundary conditions}

The periodic boundary conditions adopted in the present work are described in Pierard [30] or Herráez [31]. The displacements on two opposite faces are described by the following relations:

$$
\begin{aligned}
& \bar{u}\left(0, x_{2}, x_{3}\right)-\bar{u}\left(a, x_{2}, x_{3}\right)=\overline{u_{1}} \\
& \bar{u}\left(x_{1}, 0, x_{3}\right)-\bar{u}\left(x_{1}, b, x_{3}\right)=\overline{u_{2}} \\
& \bar{u}\left(x_{1}, x_{2}, 0\right)-\bar{u}\left(x_{1}, x_{2}, c\right)=\overline{u_{3}}
\end{aligned}
$$

Where $a, b$ and $c$ are the dimensions of the unit cell, $x_{1}, x_{2}$ and $x_{3}$ are the coordinates of any material point on the remote boundary of the RVE. $\overline{u_{1}}, \overline{u_{2}}$ and $\overline{u_{3}}$ represent the loadings applied to the reference points of the cell. $\overline{u_{1}}$ describes the displacement of the first reference point, representing the relative movement of the two faces of normal 1 . For instance, a uniaxial tension along the $x_{1}$ axis is obtained with $\overline{u_{1}}=(u, 0,0)$, $\overline{u_{2}}=\left(0, u_{2}, 0\right)$ and $\overline{u_{3}}=\left(0,0, u_{3}\right)$ where $u$ is a small displacement prescribed to the reference point. $u$ can be linked to the engineering strain $\varepsilon$ by $u=\varepsilon a$. The displacements $u_{2}$ and $u_{3}$ correspond to the lateral contractions due to Poisson's effect and computed from the condition that the force acting on surfaces having normal vectors perpendicular to the 1-axis (direction of loading here) remain free of loading (overall free surface). This PBC (B.1) applies for a unit cell periodic in the three directions of space, e.g. the 3D RVE of the yarn presented in Fig. 8a of section 3.2. In that case, the dimension of the RVE is: $a=b=c=l(J)$ where $l(J)$ is defined in Eq. (A.1). For the simulations of $2 \mathrm{D}$ periodic RVE of yarns (see Fig. $8 \mathrm{~b}$ of section 3.2), the periodic boundary conditions described by Eq. (B.1) are applied, only in the plane. In that case, we consider opposite edges instead of opposite faces. We have also $a=b=l(J)$.

The 3D RVE of the laminate displayed in Fig. 12 presents an in-plane periodicity, since the whole thickness of the laminate is considered in the numerical model. Hence PBC described by Eq. (B.1) only apply to in-plane loadings with faces of normal vector 1 or 2 . The faces of normal 3 are free surfaces. For shear or tensile loading on faces of normal vector 3 , we consider that faces with normal vector 1 or 2 are supporting PBC while the faces of normal vector 3 remain planar. In that case, as pointed out in Fig. 12, $a=2 b_{w}, b=2 b_{f}$ and $c=h$.

Since on Abaqus sofware, the meshes on two opposite faces are usually different even when the unit cell is periodic, each node on one face does not always coincide with another node on the opposite face. To overcome this problem, an exact copy of all nodes of one face has been superimposed to nodes of the opposite face. This set of new nodes has then been linked to this opposite face with a tie constraint. The constraint equation (B.1) is thus set to link the displacements of all nodes of the face and the displacements of their exact copies on the opposite face. 


\section{References}

[1] W. Kpobie, M. Martiny, S. Mercier, F. Lechleiter, L. Bodin, Thermomechanical simulation of pcb with embedded components, Microelectronics Reliability 65 (2016) 108-130.

[2] A. Salahouelhadj, M. Martiny, S. Mercier, L. Bodin, D. Manteigas, B. Stephan, Reliability of thermally stressed rigid-flex printed circuit boards for high density interconnect applications, Microelectronics Reliability 54 (2013) 204-213.

[3] K. Weinberg, W. Müller, Fatigue life and plastic deformation behaviour of electrodeposited copper thin films, Microelectronics Reliability A 527 (2010) 2222-2232.

[4] S. Huang, K. Yung, B. Sun, A finite element model and experimental analysis of PTH reliability in rigid-flex printed circuits using the taguchi method, International Journal of Fatigue 40 (2012) 84-96.

[5] F. Su, R. Mao, J. Xiong, K. Zhou, Z. Zhang, J. Shao, C. Xie, On thermo-mechanical reliability of plated-through-hole (PTH), Microelectronics Reliability 52 (2012) 1189-1196.

[6] K. Fellner, T. Antretter, P. F. Fuchs, T. Pélisset, Cyclic mechanical behavior of thin layers of copper: A theoretical and numerical study, EuroSimE (2015) 1-9.

[7] T. Ishikawa, T.-W. Chou, Stiffness and strength behaviour of woven fabric composites, Journal of Materials Science 17 (1982) 3211-3220

[8] N. K. Naik, V. K. Ganesh, Prediction of on-axes elastic properties of plain weave fabric composites, Composites Science and Technology 45 (1992) $135-152$.

[9] N. Sottos, J. Ockers, M. Swindeman, Thermoelastic properties of plain weave composites for multilayer circuit board applications, Journal of Electronic Packaging 121 (1999) 37-43.

[10] Z. Hashin, B. W. Rosen, The elastic moduli of fiber-reinforced materials, Journal of Applied Mechanics 31 (1964) 223-232.

[11] J. Xiong, R. Shenoi, J. Gao, An analytical model to predict residual thermal stress in 2D orthogonal plain weave fabric composites, International Journal of Solids and Structures 46 (2009) 1872-1883.

[12] O. Döbrich, T. Gereke, C. Cherif, Modeling the mechanical properties of textile-reinforced composites with a near micro-scale approach, Composite Structures 135 (2016) 1-7.

[13] Y. Wang, X. Sun, Digital element simulation of textile processes, Journal of Composites Science and Technology 63 (2001) 311-319.

[14] P. Fuchs, G. Pinter, M. Tonjec, Determination of the orthotropic material properties of individual layers of printed circuit boards, Microelectronics Reliability 52 (2012) 2723-2730.

[15] E. Barbero, J. Trovillion, J. Mayugo, K. Sikkil, Finite element modeling of plain weave fabrics from photomicrograph measurements, Composite Structures 73 (2006) 41-52.

[16] Z. Chen, F. Yang, S. Meguid, Multi-level modeling of woven glass/epoxy composite for multilayer printed circuit board applications, International Journal of Solids and Structures 51 (2014) 3679-3688.

[17] S. Jacques, I. De Baere, W. Van Paepegem, Application of periodic boundary conditions on multiple part finite element meshes for the mesoscale homogenization of textile fabric composites, Composites Science and Technology 92 (2014) 41-54

[18] X. Bai, M. A. Bessa, A. R. Melro, P. P. Camanho, L. Guo, W. K. Liu, High-fidelity micro-scale modeling of the thermo-visco-plastic behavior of carbon fiber polymer matrix composites, Composite Structures 134 (2015) 132-141.

[19] S. Green, M. Matveev, A. Long, D. Ivanov, S. Hallett, Mechanical modelling of $3 \mathrm{~d}$ woven composites considering realistic unit cell geometry, Composite Structures 118 (2014) 284-293.

[20] H. Srbova, T. Kroupa, V. Lukes, Comparison of homogenization approaches used for the identification of the material parameters of unidirectional composites, Materials and Technology 51 (2017) 373-378.

[21] S. G. Abaimov, A. A. Khudyakova, S. V. Lomov, On the closed form expression of the Mori-Tanaka theory prediction for the engineering constants of a unidirectional fiber-reinforced ply, Composite Structures 142 (2016) 1-6.

[22] R. M. Jones, Mechanics of Composite Materials $2^{\text {nd }}$ edition, Taylor Francis, 1998.

[23] G. Weng, The theoretical connection between Mori-Tanaka's theory and the Hashin-Strickman-Walpole bounds, International Journal of Engineering Science 28 (1990) 1111-1120.
[24] T. Mura, Micromechanics of Defects in Solids, Dordrecht: Kluwer Academic Publishers, 1987.

[25] R. Hill, Theory of mechanical properties of fiber-strengthened materials: I. elastic behavior, Journal of the Mechanics and Physics of Solids 12 (1964) 199.

[26] Z. Hashin, Viscoelastic fiber reinforced materials, AIAA Journal 4 (1966) 1411 .

[27] B. D. Lubachevsky, How to simulate billiards and similar systems, Journal of Computational Physics 94 (1991) 255-283.

[28] S. Torquato, Random Heterogeneous Materials, Springer, 2002.

[29] P. Smith, Computer simulation results for the two-point probability function of composite media, Journal of Computational Physics 76 (1988) 176-191.

[30] O. Pierard, J. LLorca, J. Segurado, I. Doghri, Micromechanics of particlereinforced elasto-viscoplastic composites: Finite element simulations versus affine homogenization, International Journal of Plasticity 23 (2007) 1041-1060.

[31] M. Herráez, C. González, C. Lopes, R. Guzmán de Villoria, J. LLorca, T. Varela, J. Sánchez, Computational micromechanics evaluation of the effect of fibre shape on the transverse strength of unidirectional composites: An approach to virtual materials design, Composites 91 (2016) 484492. 